Research Article

\title{
The Dynamics of a Tritrophic Leslie-Gower Food-Web System with the Effect of Fear
}

\author{
Firas Hussean Maghool (D) and Raid Kamel Naji \\ Department of Mathematics, College of Science, University of Baghdad, Baghdad, Iraq \\ Correspondence should be addressed to Raid Kamel Naji; rknaji@gmail.com
}

Received 19 June 2021; Revised 30 July 2021; Accepted 5 August 2021; Published 1 September 2021

Academic Editor: Oluwole D. Makinde

Copyright (c) 2021 Firas Hussean Maghool and Raid Kamel Naji. This is an open access article distributed under the Creative Commons Attribution License, which permits unrestricted use, distribution, and reproduction in any medium, provided the original work is properly cited.

\begin{abstract}
The avoidance strategy of prey to predation and the predation strategy for predators are important topics in evolutionary biology. Both prey and predators adjust their behaviors in order to obtain the maximal benefits and to raise their biomass for each. Therefore, this paper is aimed at studying the impact of prey's fear and group defense against predation on the dynamics of the food-web model. Consequently, in this paper, a mathematical model that describes a tritrophic LeslieGower food-web system is formulated. Sokol-Howell type of function response is adapted to describe the predation process due to the prey's group defensive capability. The effects of fear due to the predation process are considered in the first two levels. It is assumed that the generalist predator grows logistically using the Leslie-Gower type of growth function. All the solution properties of the model are studied. Local dynamics behaviors are investigated. The basin of attraction for each equilibrium is determined using the Lyapunov function. The conditions of persistence of the model are specified. The study of local bifurcation in the model is done. Numerical simulations are implemented to show the obtained results. It is watched that the system is wealthy in its dynamics including chaos. The fear factor works as a stabilizing factor in the system up to a specific level; otherwise, it leads to the extinction of the predator. However, increasing the prey's group defense leads to extinction in predator species.
\end{abstract}

\section{Introduction}

Mathematical modeling is used to understand the interaction of organisms with their surrounding environment, as well as species evolution that is well-known biological evolution. Nonlinear differential equation systems have always played an important role in simulating such mathematical models. Therefore, in the last few decades, there are increasing interests in the study of the dynamics of such systems. After the pioneering prey-predator model by Lotka-Volterra, the prey-predator models have been essential in mathematical ecology and have been extensively investigated. The Lotka-Volterra model was then adjusted by compiling a prey's logistic growth and a Holling-type II functional response to describe the predation [1].

It is completely recognized that a prey-predator is fundamental interaction for drawing the dynamics of food webs in the real-world environment, predation risks can negatively affect prey biomass and growth efficiency, and thus, predators affect the structure of natural communities. Prey can die of fear, and it can also reduce fertility, as a result of which fear can be just as important as outright killing by predators in affecting prey numbers [2-4]. Many prey-predator models have been suggested and investigated thoroughly in which either the predator kills the prey or the presence of the predator affects the behavior of the prey population due to the fear of the predation process [5]. Upadhyay and Mishra [6] modeled a prey-predator system taken into account the fear effect in prey growth. They watched that a relative rise in the scale of fear may minimize the size of the total population. Panday et al. [7] investigated the action of fear in a three-species food chain model, where the evolution rate of lower predator is minimized due to the fear's action from a higher predator, and the evolution rate of prey 
is reduced due to the fear's action from the lower predator. Das and Samanta [8] studied the fear's impact from the predator on prey in case of providing additional food for the predator. Recently, Kumar and Kumari [9] discussed the action of fear on the dynamics of the food chain model having three species. They obtained that for low fear's scale, the system stays chaotic while relatively high fear's scale leads to stability. Moreover, the rise of fear's scale further causes population extinction.

On the other hand, Aziz-Alaoui [10] investigated a dynamic system consisting of a Leslie-Gower food chain of three species. He displayed that for a realistic parameter set, chaotic dynamics could be obtained. Zhang et al. [11] studied a harvested Leslie-Gower prey-predator model. They got with the help of Pontryagin's maximal principle the optimal harvesting policy for the model. Cai et al. [12] investigated a Leslie-Gower prey-predator model with the diffusion and Allee effect on prey. They gained that the Allee effect basically rises the model spatiotemporal complexity. Later on, Abid et al. [13] were interested in the stability and optimal harvesting of a modified Leslie-Gowe preypredator model with Holling-type II functional response. Recently, Singh and Bhadauria [14] studied the dynamics of the prey-predator model with weak Allee effect II and modified Leslie-Gower. They explained that Allee effect II greatly impacts the model and can raise the risk of extinction.

Keeping the above in mind, in this paper, a tritrophic Leslie-Gower food-web system is constructed mathematically. Sokol-Howell type of function response [15] is used for describing the predation process due to the prey's group's defensive capability. The effects of fear due to the predation process are included and studied. The following section is written out as follows: the construction of the model is done. In Section 3, existence and local stability analysis are studied. In Section 4, persistence is discussed. However, in Section 5, the region of attraction for each equilibrium point of system (4) is determined by using the direct method of Lyapunov. Local bifurcation analysis is discussed in Section 6. In Section 7, the numerical simulation of the model is studied. Finally, Section 8 gives the conclusion.

\section{The Mathematical Construction of the Model}

In this section, a real-world tritrophic food-web system is constructed mathematically. It is supposed that the logistic law is applied for both the prey and the generalist-predator so that the environmental carrying capacity of the generalist-predator is not a constant but a function of the available food quantity so that there are upper limits to the rates of increase of both prey and generalist-predator. Therefore, the Leslie-Gower model is used in the construction of the proposed model, which assumes that both the prey and the generalist-predator grow according to the logistic law. Moreover, both the prey and specialist-predator have a defensive property against the predation when they are available in abundance. Therefore, a simplified Hollingtype IV functional response or Sokol-Howell type of func- tional response is used in the proposed model. This is because the per capita predation rate in the Sokol-Howell functional response rises with prey density to a maximum at a critical prey density beyond which it decreases.

Let $X(T)$ represents the prey density at the time $T, Y(T)$ is the specialist-predator density at the time $T$, while $Z(T)$ is the generalist-predator density at time $T$. Accordingly, the dynamics of such a food-web system can be described as follows:

$$
\begin{gathered}
\frac{d X}{d T}=X g(X)-Y P(X)-Z q_{1}(X, Y), \\
\frac{d Y}{d T}=a_{3} Y P(X)-Z q_{2}(X, Y)-d Y, \\
\frac{d Z}{d T}=c_{1}\left[Z^{2}-\frac{Z^{2}}{c_{2}+c_{3} X+c_{4} Y}\right],
\end{gathered}
$$

where $g(X)=r(1-b X)$ is the logistic growth rate of the prey species, while $P(X)=a_{0} X /\left(1+m_{0} X^{2}\right)$ and $q_{1}(X, Y)=a_{1} X /(1$ $\left.+m_{1} X^{2}+m_{2} Y^{2}\right)$, with $q_{2}(X, Y)=a_{2} X /\left(1+m_{1} X^{2}+m_{2} Y^{2}\right)$, are the Sokol-Howell functional responses for the specialistpredator and generalist-predator, respectively. The parameters of system (1) are supposed to be positive, and they are described as the following: the parameter $r>0$ represents the intrinsic growth rate, $(1 / b)>0$ is the carrying capacity of the environment for the prey, the positive parameters $a_{0}, a_{1}$, and $a_{2}$ are the maximum attack rates, the positive parameters $m_{0}, m_{1}$, and $m_{2}$ are the preference rates of the predator to their prey species, $a_{3}>0$ represents the conversion rate from individuals of the prey to specialist-predator, and $c_{1}>0$ is the intrinsic growth rate of a generalist-predator, while the positive parameters $c_{3}$ and $c_{4}$ are the respective food preference of generalistpredator from the prey and specialist-predator, respectively. In addition to the above, the square term $c_{1} Z^{2}$ in the third equation that represents the modified Leslie-Gower term signifies the fact that mating frequency is directly proportional to the number of males as well as to that of females, see $[16,17]$. Finally, the parameter $c_{2}>0$ is a constant added in the denominator to normalize the residual reduction in the generalistpredator due to a heavy lack of the preferable food $X$ and $Y$, while $d>0$ is the natural death rate of the specialist-predator. Now, to add the factor of the fear for each of the prey and the specialist-predator from the predation by an upper-level predator, the growth of each of them should be reduced by a rate proportional with the abundance of their predators. Therefore, the dynamics of the food-web system given by system (1), in case of the existence of fear, can be represented as

$$
\begin{aligned}
& \frac{d X}{d T}=\frac{r X}{\left(1+n_{1} Y+n_{2} z\right)}-b X^{2}-\frac{a_{0} X Y}{1+m_{0} X^{2}}-\frac{a_{1} X Z}{1+m_{1} X^{2}+m_{2} Y^{2}}, \\
& \frac{d Y}{d T}=\frac{a_{3} X Y}{1+m_{0} X^{2}}\left(\frac{1}{1+n_{3} Z}\right)-\frac{a_{2} Y Z}{1+m_{1} X^{2}+m_{2} Y^{2}}-d Y, \\
& \frac{d Z}{d T}=c_{1}\left[Z^{2}-\frac{Z^{2}}{c_{2}+c_{3} X+c_{4} Y}\right],
\end{aligned}
$$

where the positive parameters $n_{1}, n_{2}$, and $n_{3}$ represent the fear 
coefficients of the prey and specialist-predator from their direct predator. Clearly, system (2) has 16 parameters, which leads to analysis difficulty. So, in order to simplify the analysis of system (2) and minimize the number of parameters, the following nondimensional variables and parameters are utilized.

$$
\begin{aligned}
& x=\frac{b X}{r}, y=\frac{a_{o} Y}{r}, z=\frac{a_{o} Z}{r}, t=r T, \alpha_{1}=\frac{n_{1} r}{a_{o}}, \alpha_{2}=\frac{n_{2} r}{a_{1}}, \alpha_{3}=\frac{m_{o} r^{2}}{b^{2}} \\
& \alpha_{4}=\frac{m_{1} r^{2}}{b^{2}}, \alpha_{5}=\frac{m_{2} r^{2}}{a_{o}^{2}}, \alpha_{6}=\frac{a_{3}}{b}, \alpha_{7}=\frac{n_{3} r}{a_{1}}, \alpha_{8}=\frac{a_{o}}{a_{1}} \\
& \alpha_{9}=\frac{d}{r}, \alpha_{10}=\frac{c}{a_{1}}, \alpha_{11}=c_{2}, \alpha_{12}=\frac{c_{3} r}{b}, \alpha_{13}=\frac{c_{4} r}{a_{o}} .
\end{aligned}
$$

Therefore, the nondimensional system corresponding to system (2) is given by

$$
\begin{aligned}
& \frac{d x}{d t}=x\left[\frac{1}{1+\alpha_{1} y+\alpha_{2} z}-x-\frac{y}{1+\alpha_{3} x^{2}}-\frac{z}{1+\alpha_{4} x^{2}+\alpha_{5} y^{2}}\right]=x f_{1}, \\
& \frac{d y}{d t}=y\left[\frac{\alpha_{6} x}{1+\alpha_{3} x^{2}}\left(\frac{1}{1+\alpha_{7} z}\right)-\frac{\alpha_{8} z}{1+\alpha_{4} x^{2}+\alpha_{5} y^{2}}-\alpha_{9}\right]=y f_{2}, \\
& \frac{d z}{d t}=z\left[\alpha_{10} z-\frac{\alpha_{10} z}{\alpha_{11}+\alpha_{12} x+\alpha_{13} y}\right]=z f_{3} .
\end{aligned}
$$

Here, the interaction functions are defined on $\mathbb{R}_{+}^{3}=\{($ $x, y, z): x(t) \geq 0, y(t) \geq 0, z(t) \geq 0\}$. Moreover, since the right-hand side functions of system (4) are continuous and have continuous partial derivatives, hence they are Lipschitz functions. Thus, the solution of system (4) exists and is unique.

Theorem 1. The solutions of system (4), which start in $\mathbb{R}_{+}{ }^{3}$ are uniformly bounded.

Proof. Let $(x(t), y(t), z(t))$ be a solution of system (4) with nonnegative initial condition $\left(x_{0}, y_{0}, z_{0}\right) \in \mathbb{R}_{+}^{3}$. From the first equation in system (4), it is obtained that

$$
\frac{d x}{d t} \leq x-x^{2}
$$

hence, by solving this differential inequality, it is obtained that $x \leq 1$ as $t \longrightarrow \infty$. Define the function $w_{1}=x+\left(y / \alpha_{6}\right)$; then, the following is resulting

$$
\frac{d w_{1}}{d t} \leq \frac{1}{4}+\alpha_{9}-\alpha_{9}\left(x+\frac{y}{\alpha_{6}}\right)
$$

or $\left(d w_{1} / d t\right)+\alpha_{9} w_{1} \leq(1 / 4)+\alpha_{9}$.

Then, solving this differential inequality gives for $t \longrightarrow$ $\infty:$

$$
w_{1}=x+\frac{y}{\alpha_{6}} \leq \frac{1}{4 \alpha_{9}}+1
$$

Assume that $w_{2}=x+\left(y / \alpha_{6}\right)+z$, then by differentiating it with respect to time gives

$$
\frac{d w_{2}}{d t} \leq \frac{1}{4}+\alpha_{9}-\alpha_{9}\left(x+\frac{y}{\alpha_{6}}+z\right)+\alpha_{9} z+\alpha_{10} Z^{2}-\frac{\alpha_{10}}{\theta} Z^{2}
$$

where $\theta=\alpha_{11}+\alpha_{12}+\alpha_{13}\left(\left(\alpha_{6} / 4 \alpha_{9}\right)+\alpha_{6}\right)$.

Therefore, rearranging and substituting the upper bound of the logistic term give

$$
\frac{d w_{2}}{d t}+\alpha_{9} w_{2} \leq \frac{1}{4}+\alpha_{9}+\beta_{1}
$$

where $\beta_{1}=\alpha_{9}^{2} \theta / 4 \alpha_{10}(1-\theta)$.

Then, solving the last differential inequality as $t \longrightarrow \infty$ gives

$$
w_{2} \leq \frac{1}{4 \alpha_{9}}+1+\frac{\beta_{1}}{\alpha_{9}}=\beta_{2}
$$

Hence, all solutions of system (4) that initiate in $\mathbb{R}_{+}{ }^{3}$ are confined in the region $\Lambda=\left\{(x, y, z) \in R_{+}^{3}, w_{2}(t) \leq \beta_{2}\right\}$, that is, all the solution are uniformly bounded.

\section{Equilibria and Their Local Stability}

System (4) has at most six equilibrium points, which can be described as follows:

(1) The trivial equilibrium point (TP) denoted by $E_{0}=$ $(0,0,0)$ always exist

(2) The first axial equilibrium point (FAP) denoted by $E_{1 x}=(1,0,0)$ always exist

(3) The second axial equilibrium point (SAP) denoted by $E_{1 z}=(0,0, \widehat{z})$, where $\widehat{z}$ is any positive real number, exists if and only if

$$
\alpha_{11}=1
$$

(4) The generalist-predator free equilibrium point (GPFP) that denoted by $E_{2}=(\bar{x}, \bar{y}, 0)$, where

$$
\begin{aligned}
& \bar{x}=\frac{\alpha_{6}+\sqrt{\alpha_{6}^{2}-4 \alpha_{9}^{2} \alpha_{3}}}{2 \alpha_{9} \alpha_{3}}, \\
& \bar{y}=\frac{-\sigma_{1}(\bar{x})+\sqrt{\left[\sigma_{1}(\bar{x})\right]^{2}-4 \alpha_{1} \sigma_{0}(\bar{x})}}{2 \alpha_{1}},
\end{aligned}
$$

with $\sigma_{1}(\bar{x})=\alpha_{1} \bar{x}+\alpha_{1} \alpha_{3} \bar{x}^{3}+1>0$, and $\sigma_{0}(\bar{x})=(\bar{x}-1)[1+$ $\left.\alpha_{3} \bar{x}^{2}\right]$, exists uniquely in the positive quadrant of $x y$-plane 
if and only if the following conditions hold:

$$
\begin{gathered}
4 \alpha_{9}^{2} \alpha_{3} \leq \alpha_{6}^{2}, \\
\bar{x}<1
\end{gathered}
$$

(5) The specialist-predator free equilibrium point (SPFP) denoted by $E_{3}=(\overline{\bar{x}}, 0, \overline{\bar{z}})$ can be represented as

$$
\begin{gathered}
\overline{\bar{x}}=\frac{1-\alpha_{11}}{\alpha_{12}} \\
\overline{\bar{z}}=\frac{-\beta_{1}(\overline{\bar{x}})+\sqrt{\left[\beta_{1}(\overline{\bar{x}})\right]^{2}-4 \alpha_{2} \beta_{0}(\overline{\bar{x}})}}{2 \alpha_{2}},
\end{gathered}
$$

where $\beta_{1}(\overline{\bar{x}})=\alpha_{2} \overline{\bar{x}}+\alpha_{2} \alpha_{4} \overline{\bar{x}}^{3}+1>0$, and $\beta_{0}(\overline{\bar{x}})=(\overline{\bar{x}}-1)[1+$ $\left.\alpha_{4} \overline{\bar{x}}^{2}\right]$. Obviously, SPFP exists uniquely in the $x z$-plane if and only if the following condition holds:

$$
\alpha_{11}+\alpha_{12}>1
$$

(6) The coexistence equilibrium point (CP) denoted by $E_{4}=\left(x^{*}, y^{*}, z^{*}\right)$ can be represented as follows:

$$
x^{*}=\frac{1-\left(\alpha_{11}+\alpha_{13} y^{*}\right)}{\alpha_{12}} .
$$

The point $\left(y^{*}, z^{*}\right)$ represents the intersection point of the following two isoclines in the positive quadrant of the $y z$-plane.

$$
\begin{aligned}
& g_{1}(y, z)=\frac{1}{1+\alpha_{1} y+\alpha_{2} z}-x^{*}-\frac{y}{1+\alpha_{3} x^{* 2}}-\frac{z}{1+\alpha_{4} x^{* 2}+\alpha_{5} y^{2}}=0, \\
& g_{2}(y, z)=\frac{\alpha_{6} x^{*}}{1+\alpha_{3} x^{* 2}}\left(\frac{1}{1+\alpha_{7} z}\right)-\frac{\alpha_{8} z}{1+\alpha_{4} x^{* 2}+\alpha_{5} y^{2}}-\alpha_{9}=0 .
\end{aligned}
$$

Simple computation displays that the two isoclines given by (16a) and (16b) have a unique positive intersection point, denoted by $\left(y^{*}, z^{*}\right)$, provided that the following sufficient conditions hold:

$$
\begin{gathered}
\alpha_{11}+\alpha_{13} y^{*}<1, \\
\alpha_{11}+\alpha_{12}<1, \\
\alpha_{9} \alpha_{12}^{2}+\alpha_{9} \alpha_{3}\left(1-\alpha_{11}\right)^{2}<\alpha_{12} \alpha_{6}\left(1-\alpha_{11}\right), \\
y_{1}<y_{2},
\end{gathered}
$$

$$
\frac{d y}{d z}=-\frac{\left(\partial g_{1} / \partial z\right)}{\left(\partial g_{1} / \partial y\right)}>0,
$$

$$
\frac{d y}{d z}=-\frac{\left(\partial g_{2} / \partial z\right)}{\left(\partial g_{2} / \partial y\right)}<0
$$

Here, $y_{1}$ and $y_{2}$ represent the positive root of the following two polynomials, respectively:

$$
\begin{gathered}
\varsigma_{1} y^{4}+\varsigma_{2} y^{3}+\varsigma_{3} y^{2}+\varsigma_{4} y+\varsigma_{5}=0, \\
\varsigma_{6} y^{2}+\varsigma_{7} y+\varsigma_{8}=0,
\end{gathered}
$$

where

$\varsigma_{1}=\alpha_{1} \alpha_{3} \alpha_{13}^{3}>0$,

$\varsigma_{2}=\alpha_{3} \alpha_{13}^{2}\left(\alpha_{13}-3 \alpha_{1}\left(1-\alpha_{11}\right)\right)>0$,

$\varsigma_{3}=\alpha_{1} \alpha_{12}^{2}\left(\alpha_{13}-\alpha_{12}\right)+3 \alpha_{13} \alpha_{3}\left(1-\alpha_{11}\right)\left[\alpha_{1}\left(1-\alpha_{11}\right)-\alpha_{13}\right]+\alpha_{3} \alpha_{13}^{2} \alpha_{12}>0$,

$\varsigma_{4}=\alpha_{12}^{2}\left(\alpha_{1} \alpha_{11}+\alpha_{13}-\alpha_{1}\right)+\alpha_{3}\left(1-\alpha_{11}\right)^{2}\left(\alpha_{1} \alpha_{11}+\alpha_{13}-\alpha_{1}\right)$

$-2 \alpha_{3} \alpha_{13}\left(1-\alpha_{11}\right)\left(\alpha_{12}+\alpha_{11}-1\right)-\alpha_{12}^{3}$,

$\varsigma_{5}=\left[\alpha_{12}-\left(1-\alpha_{11}\right)\right]\left[\alpha_{12}^{2}+\left(1-\alpha_{11}\right)^{2}\right]<0$,

$\varsigma_{6}=\alpha_{9} \alpha_{3} \alpha_{12}^{2}>0$

$\varsigma_{7}=\alpha_{12} \alpha_{9} \alpha_{13}-2 \alpha_{9} \alpha_{3} \alpha_{13}\left(1-\alpha_{11}\right)$

$\varsigma_{8}=\alpha_{9} \alpha_{12}^{2}+\alpha_{9} \alpha_{3}\left(1-\alpha_{11}\right)^{2}+\alpha_{11} \alpha_{12} \alpha_{6}-\alpha_{12} \alpha_{6}<0$.

Now, the local stability of the above equilibrium points is studied by computing the Jacobian matrix (JM) and then determining their eigenvalues. It is simply to verify that the JM of system (4) at the point $(x, y, z)$ can be represented as

$$
J=\left(\begin{array}{ccc}
x \frac{\partial f_{1}}{\partial x}+f_{1} & x \frac{\partial f_{1}}{\partial y} & x \frac{\partial f_{1}}{\partial z} \\
y \frac{\partial f_{2}}{\partial x} & y \frac{\partial f_{2}}{\partial y}+f_{2} & y \frac{\partial f_{2}}{\partial z} \\
z^{2} \frac{\partial f_{3}}{\partial x} & z^{2} \frac{\partial f_{3}}{\partial y} & z^{2} \frac{\partial f_{3}}{\partial z}+2 z f_{3}
\end{array}\right),
$$


where

$$
\begin{gathered}
\frac{\partial f_{1}}{\partial x}=-1+\frac{2 \alpha_{3} x y}{\Gamma_{2}{ }^{2}}+\frac{2 \alpha_{4} x z}{\Gamma_{1}{ }^{2}}, \\
\frac{\partial f_{1}}{\partial y}=-\frac{\alpha_{1}}{\Gamma_{3}^{2}}-\frac{1}{\Gamma_{2}}+\frac{2 \alpha_{5} y z}{\Gamma_{1}^{2}}, \\
\frac{\partial f_{1}}{\partial z}=-\frac{\alpha_{2}}{\Gamma_{3}^{2}}-\frac{1}{\Gamma_{1}}, \\
\frac{\partial f_{2}}{\partial x}=\frac{\alpha_{6}-\alpha_{6} \alpha_{3} x^{2}}{\Gamma_{2}^{2} \Gamma_{4}}+\frac{2 \alpha_{8} \alpha_{4} x z}{\Gamma_{1}^{2}}, \\
\frac{\partial f_{2}}{\partial y}=\frac{2 \alpha_{5} \alpha_{8} y z}{\Gamma_{1}^{2}}, \\
\frac{\partial f_{2}}{\partial z}=-\frac{\alpha_{6} \alpha_{7} x}{\Gamma_{2} \Gamma_{4}^{2}}-\frac{\alpha_{8}}{\Gamma_{1}}, \\
\frac{\partial f_{3}}{\partial x}=\frac{\alpha_{10} \alpha_{12}}{\Gamma_{5}^{2}}, \\
\frac{\partial f_{3}}{\partial y}=\frac{\alpha_{10} \alpha_{13}}{\Gamma_{5}^{2}}, \\
\frac{\partial f_{3}}{\partial z}=0,
\end{gathered}
$$

with $\Gamma_{1}=1+\alpha_{4} x^{2}+\alpha_{5} y^{2}, \Gamma_{2}=1+\alpha_{3} x^{2}, \Gamma_{3}=1+\alpha_{1} y+\alpha_{2} z$, $\Gamma_{4}=1+\alpha_{7} z$, and $\Gamma_{5}=\alpha_{11}+\alpha_{12} x+\alpha_{13} y$.

Consequently, for the TP, the JM becomes

$$
J_{E_{0}}=\left(\begin{array}{ccc}
1 & 0 & 0 \\
0 & -\alpha_{9} & 0 \\
0 & 0 & 0
\end{array}\right) .
$$

Then, the eigenvalues of $J_{E_{0}}$ are given by $\lambda_{10}=1>0$, $\lambda_{20}=-\alpha_{9}<0$, and $\lambda_{30}=0$. Since there is a positive equilibrium point, then $E_{0}$ is an unstable nonhyperbolic point.

For the FAP, the JM can be represented as

$$
J_{E_{1 x}}=\left(\begin{array}{ccc}
-1 & 0 & 0 \\
0 & \frac{\alpha_{6}}{1+\alpha_{3}}-\alpha_{9} & 0 \\
0 & 0 & 0
\end{array}\right) \text {. }
$$

Then, the eigenvalues of $J_{E_{1 x}}$ are clearly given by $\lambda_{11}=-$ $1<0, \lambda_{21}=\left(\alpha_{6} /\left(1+\alpha_{3}\right)\right)-\alpha_{9}$, and $\lambda_{31}=0$. Hence, the FAP is a nonhyperbolic point due to the existence of zero eigenvalue.

For the SAP, the JM can be represented as

$$
J_{E_{1 z}}=\left(\begin{array}{ccc}
\frac{1}{1+\alpha_{2} \widehat{z}}-\widehat{z} & 0 & 0 \\
0 & -\left(\alpha_{8} \widehat{z}+\alpha_{9}\right) & 0 \\
\frac{\alpha_{10} \alpha_{12} \widehat{z}^{2}}{\alpha_{11}{ }^{2}} & \frac{\alpha_{10} \alpha_{13} \hat{z}^{2}}{\alpha_{11}{ }^{2}} & 0
\end{array}\right) .
$$

Then, the eigenvalues of $J_{E_{1 z}}$ are clearly given by $\lambda_{21}=(1 /$ $\left.\left(1+\alpha_{2} \widehat{z}\right)\right)-\widehat{z}, \lambda_{22}=-\left(\alpha_{8} \widehat{z}+\alpha_{9}\right)<0$, and $\lambda_{23}=0$. Hence, the SAP is a nonhyperbolic point due to the existence of zero eigenvalue.

For the GPFP, the JM can be represented as

$$
J_{E_{2}}=\left[a_{i j}\right]_{3 \times 3},
$$

where

$$
\begin{gathered}
a_{11}=\bar{x}_{1}\left[-1+\frac{2 \alpha_{3} \bar{x}_{1} \bar{y}_{1}}{\bar{\Gamma}_{2}^{2}}\right], \\
a_{12}=\bar{x}_{1}\left[-\frac{\alpha_{1}}{\bar{\Gamma}_{3}^{2}}-\frac{1}{\bar{\Gamma}_{2}}\right], \\
a_{13}=\bar{x}_{1}\left[-\frac{\alpha_{2}}{\bar{\Gamma}_{3}^{2}}-\frac{1}{\bar{\Gamma}_{1}}\right], \\
a_{21}=\frac{\alpha_{6} \bar{y}_{1}\left(1-\alpha_{3} \bar{x}_{1}^{2}\right)}{\bar{\Gamma}_{2}^{2}}, \\
a_{22}=0, \\
a_{23}=\bar{x}_{1}\left[-\frac{\alpha_{6} \alpha_{7} \bar{y}_{1}}{\bar{\Gamma}_{2}^{2}}-\frac{\alpha_{8}}{\bar{\Gamma}_{1}}\right], \\
a_{31}=0, \\
a_{32}=0, \\
a_{33}=0,
\end{gathered}
$$

with $\bar{\Gamma}_{1}=1+\alpha_{4} \bar{x}_{1}^{2}+\alpha_{5} \bar{y}_{1}^{2}, \bar{\Gamma}_{2}=1+\alpha_{3} \bar{x}_{1}^{2}$, and $\bar{\Gamma}_{3}=1+\alpha_{1} \bar{y}_{1}$. Clearly, the characteristic equation of $J_{E_{2}}$ can be represented as

$$
\lambda\left[\lambda^{2}-a_{11} \lambda-a_{12} a_{21}\right]=0 .
$$

Because the JM has a zero eigenvalue $(\lambda=0)$, then $E_{2}$ is a nonhyperbolic equilibrium point.

For the SPEP, the JM can be determined as

$$
J_{E_{3}}=\left[b_{i j}\right]_{3 \times 3},
$$

where

$$
\begin{gathered}
b_{11}=\overline{\bar{x}}\left[-1+\frac{2 \alpha_{4} \overline{\bar{x}} \overline{\bar{z}}}{\overline{\bar{\Gamma}}_{1}^{2}}\right], \\
b_{12}=\overline{\bar{x}}\left[-\frac{\alpha_{1}}{\overline{\bar{\Gamma}}_{3}^{2}}-\frac{1}{\overline{\bar{\Gamma}}_{2}}\right], \\
b_{13}=\overline{\bar{x}}\left[-\frac{\alpha_{2}}{\overline{\bar{\Gamma}}_{3}^{2}}-\frac{1}{\overline{\bar{\Gamma}}_{1}}\right], \\
b_{21}=0,
\end{gathered}
$$




$$
\begin{gathered}
b_{22}=\frac{\alpha_{6} \overline{\bar{x}}}{\overline{\bar{\Gamma}}_{1}^{2} \overline{\bar{\Gamma}}_{4}}-\frac{\alpha_{8} \overline{\bar{z}}}{\overline{\bar{\Gamma}}_{1}}-\alpha_{9}, \\
b_{23}=0, \\
b_{31}=\frac{\alpha_{10} \alpha_{12} \overline{\bar{z}}^{2}}{\overline{\bar{\Gamma}}_{5}^{2}}, \\
b_{32}=\frac{\alpha_{10} \alpha_{13} \overline{\bar{z}}^{2}}{\overline{\bar{\Gamma}}_{5}^{2}}, \\
b_{33}=0
\end{gathered}
$$

with $\overline{\bar{\Gamma}}_{1}=1+\alpha_{4} \overline{\bar{x}}^{2}, \overline{\bar{\Gamma}}_{2}=1+\alpha_{3} \overline{\bar{x}}^{2}, \overline{\bar{\Gamma}}_{3}=1+\alpha_{2} \overline{\bar{z}}, \overline{\bar{\Gamma}}_{4}=1+\alpha_{7} \overline{\bar{z}}$, and $\overline{\bar{\Gamma}}_{5}=\alpha_{11}+\alpha_{12} \overline{\bar{x}}$. Therefore, the characteristic equation of $J_{E_{3}}$ can be represented as

$$
\left(b_{22}-\lambda\right)\left[\lambda^{2}-b_{11} \lambda-b_{13} b_{31}\right]=0
$$

Clearly, the roots (eigenvalues) of equation (30) can be represented as

$$
\begin{gathered}
\lambda_{13}=\frac{b_{11}+\sqrt{b_{11}^{2}-4 b_{13} b_{31}}}{2}, \\
\lambda_{23}=\frac{\alpha_{6} \overline{\bar{x}}}{\overline{\bar{\Gamma}}_{1}^{2} \overline{\bar{\Gamma}}_{4}}-\frac{\alpha_{8} \overline{\bar{z}}}{\overline{\bar{\Gamma}}_{1}}-\alpha_{9}, \\
\lambda_{33}=\frac{b_{11}-\sqrt{b_{11}^{2}-4 b_{13} b_{31}}}{2} .
\end{gathered}
$$

It is easy to verify that all the eigenvalues of $J_{E_{3}}$ are negative, and hence, the SPFP is locally asymptotically stable (L.A.S), presuming that the next conditions hold:

$$
\begin{gathered}
\frac{2 \alpha_{4} \overline{\bar{x}} \overline{\bar{z}}}{\overline{\bar{\Gamma}}_{1}^{2}}<1, \\
\frac{\alpha_{6} \overline{\bar{x}}}{\overline{\bar{\Gamma}}_{1}^{2} \overline{\bar{\Gamma}}_{4}}-\frac{\alpha_{8} \overline{\bar{z}}}{\overline{\bar{\Gamma}}_{1}}-\alpha_{9}<0 .
\end{gathered}
$$

The JM of system (4) at the CP can be represented as

$$
J_{E_{4}}=\left[c_{i j}\right]_{3 \times 3},
$$

where

$$
\begin{aligned}
& c_{11}=x^{*}\left[-1+\frac{2 \alpha_{3} x^{*} y^{*}}{\Gamma_{2 *}^{2}}+\frac{2 \alpha_{4} x^{*} z^{*}}{\Gamma_{1 *}^{2}}\right] \text {, } \\
& c_{12}=x^{*}\left[-\frac{\alpha_{1}}{\Gamma_{3 *}^{2}}-\frac{1}{\Gamma_{2 *}}+\frac{2 \alpha_{5} y^{*} z^{*}}{\Gamma_{1 *}^{2}}\right] \text {, } \\
& c_{13}=x^{*}\left[-\frac{\alpha_{2}}{\Gamma_{3 *}^{2}}-\frac{1}{\Gamma_{1 *}}\right] \text {, } \\
& c_{21}=y^{*}\left[\frac{\alpha_{6}\left(1-\alpha_{3} x^{* 2}\right)}{\Gamma_{2 *}^{2} \Gamma_{4 *}}+\frac{2 \alpha_{8} \alpha_{4} x^{*} z^{*}}{\Gamma_{1 *}^{2}}\right] \text {, } \\
& c_{22}=\frac{2 \alpha_{5} \alpha_{8} y^{* 2} z^{*}}{\Gamma_{1 *}^{2}}, \\
& c_{23}=y^{*}\left[-\frac{\alpha_{6} \alpha_{7} x^{*}}{\Gamma_{2 *} \Gamma_{4 *}^{2}}-\frac{\alpha_{8}}{\Gamma_{1 *}}\right] \text {, } \\
& c_{31}=\frac{\alpha_{10} \alpha_{12} z^{* 2}}{\Gamma_{5 *}^{2}} \text {, } \\
& c_{32}=\frac{\alpha_{10} \alpha_{13} z^{* 2}}{\Gamma_{5 *}^{2}}, \\
& c_{33}=0 \text {, }
\end{aligned}
$$

with $\Gamma_{1 *}=1+\alpha_{4} x^{* 2}+\alpha_{5} y^{* 2}, \Gamma_{2 *}=1+\alpha_{3} x^{* 2}, \Gamma_{3 *}=1+\alpha_{1}$ $y^{*}+\alpha_{2} z^{*}, \quad \Gamma_{4 *}=1+\alpha_{7} z^{*}, \quad$ and $\Gamma_{5 *}=\alpha_{11}+\alpha_{12} x^{*}+\alpha_{13} y^{*}$. Consequently, the characteristic equation of $J_{E_{4}}$ can be represented as

$$
\lambda^{3}+A_{1} \lambda^{2}+A_{2} \lambda+A_{3}=0
$$

where $A_{1}=-\left(c_{11}+c_{22}\right)$,

$$
\begin{gathered}
A_{2}=-\left(c_{13} c_{31}-c_{11} c_{22}+c_{23} c_{32}+c_{12} c_{21}\right), \\
A_{3}=-\left[c_{13}\left(c_{21} c_{32}-c_{22} c_{31}\right)+c_{23}\left(c_{12} c_{31}-c_{11} c_{32}\right)\right],
\end{gathered}
$$

with

$$
\begin{aligned}
\Delta= & A_{1} A_{2}-A_{3}=\left(c_{11}+c_{22}\right)\left[c_{11} c_{22}-c_{12} c_{21}\right] \\
& +c_{13}\left[c_{11} c_{31}+c_{21} c_{32}\right]+c_{23}\left[c_{22} c_{32}+c_{12} c_{31}\right] .
\end{aligned}
$$

A reminder that according to the Routh-Hurwitz criterion, the characteristic equation (35) has negative real part eigenvalues, and then, the CP becomes L.A.S if and only if $A_{1}>0, A_{3}>0$, and $\Delta>0$. Accordingly, the following theorem can be proved simply.

Theorem 2. The CP is L.A.S if the below sufficient conditions hold.

$$
2 \alpha_{3} x^{* 2} y^{*} \Gamma_{1 *}{ }^{2}+2 \alpha_{4} x^{* 2} z^{*} \Gamma_{2 *}{ }^{2}+2 \alpha_{5} \alpha_{8} y^{* 2} z^{*} \Gamma_{2 *}{ }^{2}<x^{*} \Gamma_{1 *}^{2} \Gamma_{2 *}^{2},
$$

$$
2 \alpha_{5} y^{*} z^{*} \Gamma_{2 *} \Gamma_{3 *}^{2}<\alpha_{1} \Gamma_{1 *}^{2} \Gamma_{2 *}+\Gamma_{1 *}^{2} \Gamma_{3 *}^{2}
$$




$$
\begin{gathered}
\alpha_{3} x^{* 2}<1, \\
\frac{c_{22}}{c_{21}}<\frac{\alpha_{13}}{\alpha_{12}}<\max \left\{\frac{c_{12}}{c_{11}}, \frac{-c_{11}}{c_{21}}, \frac{-c_{12}}{c_{22}}\right\}, \\
{\left[\frac{\left(\alpha_{1} \Gamma_{1 *}^{2} \Gamma_{2 *}+\Gamma_{1 *}^{2} \Gamma_{3 *}^{2}-2 \alpha_{5} y^{*} z^{*} \Gamma_{2 *} \Gamma_{3 *}^{2}\right)\left(\alpha_{6}\left(1-\alpha_{3} x^{*}\right) \Gamma_{1 *}^{2}+2 \alpha_{8} \alpha_{4} x^{*} z^{*} \Gamma_{2 *}^{2} \Gamma_{4 *}\right)}{\Gamma_{2 *} \Gamma_{* *}^{2} T_{4 *}\left(\Gamma_{1 *}^{2} \Gamma_{2 *}^{2}-2 \alpha_{3} x^{*} y^{*} \Gamma_{1 *}^{2}-2 \alpha_{4} x^{*} z^{*} \Gamma_{2 *}^{2}\right) \alpha_{5} \alpha_{8} y^{z^{*}} z^{*}}\right]<2 .}
\end{gathered}
$$

\section{Persistence}

In this part, the persistence of system (4) is investigated; it is fully recognized that the system is said to persist if and only if none of their species extinct; this means that system (4) persists if the trajectory of the system that initiates at a positive initial point does not have omega limit set on the boundary planes of its domain.

System (4) has two subsystems lying in $x y$-plane and $x z$ -plane, respectively, which can be represented as follows:

$$
\begin{gathered}
\frac{d x}{d t}=x\left[\frac{1}{1+\alpha_{1} y}-x-\frac{y}{1+\alpha_{3} x^{2}}\right]=h_{1}(x, y), \\
\frac{d y}{d t}=y\left[\frac{\alpha_{6} x}{1+\alpha_{3} x^{2}}-\alpha_{9}\right]=h_{2}(x, y),
\end{gathered}
$$

and

$$
\begin{gathered}
\frac{d x}{d t}=x\left[\frac{1}{1+\alpha_{2} z}-x-\frac{z}{1+\alpha_{4} x^{2}}\right]=G_{1}(x, z), \\
\frac{d z}{d t}=Z^{2}\left[\alpha_{10}-\frac{\alpha_{10}}{\alpha_{11}+\alpha_{12} x}\right]=G_{2}(x, z) .
\end{gathered}
$$

It can be simply confirmed that the above subsystems (39) and (40) have positive equilibrium points that coincide with those of system (4) in the interior of boundary planes $x y$-plane and $x z$-plane, respectively. Now, to discover the possibility of the existence of periodic dynamics in the boundary planes, the Dulac function approach is used.

Consider the following function $B_{1}(x, y)=1 / x y$, clearly this function $B_{1}(x, y)>0$ and $C^{1}$ function in the interior of $\mathbb{R}_{+}^{2}$ of $x y$-plane. Moreover, it is obvious that

$$
\Delta(x, y)=\frac{\partial}{\partial x}\left(B_{1} \bullet h_{1}\right)+\frac{\partial}{\partial y}\left(B_{1} \bullet h_{2}\right)=-\frac{1}{y}+\frac{2 \alpha_{3} x}{\left(1+\alpha_{3} x^{2}\right)^{2}} .
$$

Therefore, $\Delta(x, y)$ does not alter sign and not vanish under the following condition:

$$
\frac{2 \alpha_{3} x}{\left(1+\alpha_{3} x^{2}\right)^{2}}<\frac{1}{y}
$$

Hence, if condition (42) holds, there are no periodic dynamics in the interior of a positive quadrant of $x y$-plane for subsystem (39).
Similarly, with the help of Dulac function $B_{2}(x, z)=1 / x$ $z^{2}$, which is $B_{2}(x, z)>0$, and $C^{1}$ function in the interior of $\mathbb{R}_{+}^{2}$ of $x z$-plane, it is observed that there are no periodic dynamics in the interior of a positive quadrant of $x z$-plane for subsystem (40) provided that the following condition holds:

$$
\frac{2 \alpha_{4} x}{\left(1+\alpha_{4} x^{2}\right)^{2}}<\frac{1}{z}
$$

Theorem 3. Suppose that the boundary planes have no periodic dynamics; then, system (4) is uniformly persistent as long as that the next conditions hold:

$$
\begin{gathered}
\alpha_{9}<\frac{\alpha_{6}}{1+\alpha_{3}}, \\
\frac{1}{\alpha_{11}+\alpha_{12}}<1, \\
\widehat{z}\left(1+\alpha_{2} \bar{z}\right)<1, \\
\frac{1}{\alpha_{11}+\alpha_{12} \bar{x}+\alpha_{13} \bar{y}}<1,
\end{gathered}
$$$$
\frac{\alpha_{8} \overline{\bar{x}}}{1+\alpha_{4} \overline{\bar{x}}^{2}}+\alpha_{9}<\frac{\alpha_{6} \overline{\bar{x}}}{1+\alpha_{3} \overline{\bar{x}}^{2}}\left(\frac{1}{1+\alpha_{7} \overline{\bar{z}}}\right) \text {. }
$$

Proof. Define a function $\vartheta(x, y, z)=x^{p_{1}} y^{p_{2}} z^{p_{3}}$, where $p_{1}, p_{2}$, and $p_{3}$ are positive constants, and $\vartheta(x, y, z)>0$ for all $(x, y$, $z) \in \operatorname{Int} \mathbb{R}_{+}^{3}$ with $9(x, y, z)=0$ if any one of $x, y$, or $z$ approaches zero. Therefore, direct computation gives

$$
\Omega(x, y, z)=\frac{\vartheta^{\prime}(x, y, z)}{\vartheta(x, y, z)}=p_{1} f_{1}+p_{2} f_{2}+p_{3} f_{3},
$$

where the functions $f_{i} ; i=1,2,3$ are given in system (4). Now, according to the average Lyapunov method, the proof is satisfied provided that $\Omega(x, y, z)>0$ for all boundary equilibrium points. Therefore,

$$
\begin{aligned}
\Omega(x, y, z)= & p_{1}\left[\frac{1}{\Gamma_{3}}-x-\frac{y}{\Gamma_{2}}-\frac{z}{\Gamma_{1}}\right]+p_{2}\left[\frac{\alpha_{6} x}{\Gamma_{2} \Gamma_{4}}-\frac{\alpha_{8} z}{\Gamma_{1}}-\alpha_{9}\right] \\
& +p_{3}\left[\alpha_{10}-\frac{\alpha_{10}}{\Gamma_{5}}\right],
\end{aligned}
$$

where $\Gamma_{i} ; i=1,2,3,4,5$ are given in equation (20). Clearly, we have that

$$
\Omega\left(E_{0}\right)=p_{1}[1]+p_{2}\left[-\alpha_{9}\right] .
$$

Obviously, by choosing the arbitrary positive value of $p_{1}$ sufficiently larger than that of $p_{2}$, it is obtained that $\Omega\left(E_{0}\right)>0$. 


$$
\Omega\left(E_{1 x}\right)=p_{2}\left[\frac{\alpha_{6}}{1+\alpha_{3}}-\alpha_{9}\right]+p_{3}\left[\alpha_{10}-\frac{\alpha_{10}}{\alpha_{11}+\alpha_{12}}\right]
$$

Note that conditions (44a) and (44b) guarantee that $\Omega\left(E_{1 x}\right)>0$.

$$
\Omega\left(E_{1 z}\right)=p_{1}\left[\frac{1}{1+\alpha_{2} \widehat{z}}-\widehat{z}\right]+p_{2}\left[-\left(\alpha_{8} \widehat{z}+\alpha_{9}\right)\right] .
$$

Again, by choosing the arbitrary positive value of $p_{1}$ sufficiently larger than that of $p_{2}$, it is obtained that $\Omega$ ( $\left.E_{1 z}\right)>0$ provided that condition $(44 \mathrm{c})$ holds.

$$
\Omega\left(E_{2}\right)=p_{3}\left[\alpha_{10}-\frac{\alpha_{10}}{\alpha_{11}+\alpha_{12} \bar{x}+\alpha_{13} \bar{y}}\right] .
$$

Clearly, condition (44d) guarantees that $\Omega\left(E_{2}\right)>0$.

$$
\Omega\left(E_{3}\right)=p_{2}\left[\frac{\alpha_{6} \overline{\bar{x}}}{1+\alpha_{3} \overline{\bar{x}}^{2}}\left(\frac{1}{1+\alpha_{7} \overline{\bar{z}}}\right)-\frac{\alpha_{8} \overline{\bar{x}}}{1+\alpha_{4} \overline{\bar{x}}^{2}}-\alpha_{9}\right] .
$$

Finally, $\Omega\left(E_{3}\right)>0$ under condition (44e). Hence, system (4) is uniformly persistent, and the proof is done.

\section{Region of Attraction}

In this section, the region of attraction for each equilibrium point of system (4) is determined using the direct method of Lyapunov. These regions of attraction for each of their equilibrium point are known as the domain or basin of attraction.

Theorem 4. The FAP is asymptotically stable (A.S) in the subregion of $\mathbb{R}_{+}^{3}$ that satisfies the following sufficient conditions:

$$
\begin{gathered}
1<\alpha_{9}, \\
\alpha_{1}<\frac{\left(1-\alpha_{6}\right)}{1+\alpha_{3}}, \\
\alpha_{2}<\frac{1}{1+\alpha_{4}+\alpha_{5}\left(\left(\alpha_{6} / 4 \alpha_{9}\right)+\alpha_{6}\right)^{2}}, \\
\frac{1}{4 \alpha_{10} G\left(\alpha_{9}-1\right)}<y,
\end{gathered}
$$

where the symbol $G$ is given in the proof.

Proof. Define $L_{1}(x, y, z)=\int_{1}^{x}((u-1) / u) d u+y+z$. Clearly, the function $L_{1}(x, y, z)$ is a positive definite function that is $L_{1}(1,0,0)=0$, while $L_{1}(x, y, z)>0$, for all values in the region $\left\{(x, y, z) \in \mathbb{R}_{+}^{3}: x>0, y \geq 0, z \geq 0 ;(x, y, z) \neq(1,0,0)\right\}$
. Then, using some algebraic manipulation gives that

$$
\begin{aligned}
\frac{d L_{1}}{d t} \leq & -\frac{(x-1)^{2}}{1+\alpha_{1} y+\alpha_{2} z}+\frac{x\left(\alpha_{1} y+\alpha_{2} z\right)}{1+\alpha_{1} y+\alpha_{2} z}-\frac{x y}{1+\alpha_{3} x^{2}}+\frac{y}{1+\alpha_{3} x^{2}} \\
& -\frac{x z}{1+\alpha_{4} x^{2}+\alpha_{5} y^{2}}+\frac{z}{1+\alpha_{4} x^{2}+\alpha_{5} y^{2}} \\
& +\frac{\alpha_{6} x y}{1+\alpha_{3} x^{2}}\left(\frac{1}{1+\alpha_{7} z}\right)-\alpha_{9} y \\
& +\alpha_{10}\left[z^{2}-\frac{Z^{2}}{\alpha_{11}+\alpha_{12} x+\alpha_{13} y}\right] .
\end{aligned}
$$

Consequently, by using additional computation, the following is obtained:

$$
\begin{aligned}
\frac{d L_{1}}{d t} \leq & -\frac{(x-1)^{2}}{1+\alpha_{1} y+\alpha_{2} z}+\alpha_{1} x y+\alpha_{2} x z-\left(\alpha_{9}-1\right) y-\frac{\left(1-\alpha_{6}\right) x y}{1+\alpha_{3} x^{2}} \\
& -\frac{x z}{1+\alpha_{4} x^{2}+\alpha_{5} y^{2}}+z-\alpha_{10} z^{2}\left[\frac{1}{\alpha_{11}+\alpha_{12} x+\alpha_{13} y}-1\right] .
\end{aligned}
$$

Now, according to the upper bound for $x$ and $y$, so as $t$ $\longrightarrow \infty$, we have $x \leq 1$ and $y \leq\left(\alpha_{6} / 4 \alpha_{9}\right)+\alpha_{6}$. Therefore, it is obtained that

$$
\begin{aligned}
\frac{d L_{1} \leq}{d t} \leq & -\frac{(x-1)^{2}}{1+\alpha_{1} y+\alpha_{2} z}-\left(\alpha_{9}-1\right) y-\left[\frac{\left(1-\alpha_{6}\right)}{1+\alpha_{3}}-\alpha_{1}\right] x y \\
& -\left[\frac{1}{1+\alpha_{4}+\alpha_{5}\left(\left(\alpha_{6} / 4 \alpha_{9}\right)+\alpha_{6}\right)^{2}}-\alpha_{2}\right] x z \\
& +z\left[1-\alpha_{10} z\left(\frac{1}{\alpha_{11}+\alpha_{12}+\alpha_{13}\left(\left(\alpha_{6} / 4 \alpha_{9}\right)+\alpha_{6}\right)}-1\right)\right] .
\end{aligned}
$$

Consequently, using conditions (52b) and (52c) with the bound of logistic term yields

$$
\begin{aligned}
\frac{d L_{1}}{d t} \leq & -\frac{(x-1)^{2}}{1+\alpha_{1} y+\alpha_{2} z}-\left(\alpha_{9}-1\right) y-\left[\frac{\left(1-\alpha_{6}\right)}{1+\alpha_{3}}-\alpha_{1}\right] x y \\
& -\left[\frac{1}{1+\alpha_{4}+\alpha_{5}\left(\left(\alpha_{6} / 4 \alpha_{9}\right)+\alpha_{6}\right)^{2}}-\alpha_{2}\right] x z+\frac{1}{4 \alpha_{10} G},
\end{aligned}
$$

where $G=1 /\left(\alpha_{11}+\alpha_{12}+\alpha_{13}\left(\alpha_{6} / 4 \alpha_{9}+\alpha_{6}\right)\right)-1$.

Accordingly, condition (52d) guarantees that $\left(d L_{1} / d t\right)$ $<0$, which means it is a negative definite. Then, $L_{1}$ is a Lyapunov function, and hence, the FAP is A.S for any trajectory starting from a point that belongs to the region that satisfies the above conditions.

Note that the subregion described in Theorem 4 represents the basin of attraction of the FAP. 
Theorem 5. The SAP is A.S in the subregion of $\mathbb{R}_{+}{ }^{3}$ that satisfies the following sufficient conditions:

$$
\begin{gathered}
\widehat{z}<\min \left\{\frac{\alpha_{11} \Gamma_{5}}{\alpha_{10} \alpha_{12} \Gamma_{1}}, \frac{\alpha_{8} \alpha_{11} \Gamma_{5}}{\alpha_{10} \alpha_{13} \Gamma_{1}}\right\}, \\
x+\alpha_{10} N(z-z \wedge)^{2}<\alpha_{9} y,
\end{gathered}
$$

where the symbol $N>0$ is given in the proof.

Proof. Define $L_{2}(x, y, z)=x+y+\int_{\widehat{z}}^{z}((m-\widehat{z}) / z) d m$. Clearly, the function $L_{2}(x, y, z)$ is a positive definite function that is $L_{2}(0,0, \widehat{z})=0$, while $L_{2}(x, y, z)>0$, for all values in the region $\left\{(x, y, z) \in \mathbb{R}_{+}^{3}: x \geq 0, y \geq 0, z>0 ;(x, y, z) \neq(0,0, \widehat{z})\right\}$ . Then, using some algebraic manipulation gives that

$$
\begin{aligned}
\frac{d L_{2}}{d t} \leq & x-x z\left(\frac{1}{\Gamma_{1}}-\frac{\alpha_{10} \alpha_{12} \widehat{z}}{\alpha_{11} \Gamma_{5}}\right)-y z\left(\frac{\alpha_{8}}{\Gamma_{1}}-\frac{\alpha_{10} \alpha_{13} \widehat{z}}{\alpha_{11} \Gamma_{5}}\right) \\
& -\alpha_{9} y+\alpha_{10} N(z-z \wedge)^{2}
\end{aligned}
$$

where $N=1-\left(1 /\left(\alpha_{11}+\alpha_{12} x+\alpha_{13} y\right)\right)$; clearly, $N$ is positive at the SAP.

Consequently, by using conditions (57a) and (57b), it is obtained that $\left(d L_{2} / d t\right)<0$. Hence, the derivative of the function $L_{2}$ is a negative definite, and then, $L_{2}$ is a Lyapunov function. Hence, the SAP becomes A.S point for any point that belongs to the subregion that satisfies the given conditions and the proof is done.

Theorem 6. The GPFP is A.S in the subregion of $\mathbb{R}_{+}{ }^{3}$ that satisfies the following sufficient conditions:

$$
\begin{gathered}
\frac{\alpha_{3} \bar{y}(x+\bar{x})}{\Gamma_{2} \bar{\Gamma}_{2}}<1, \\
\Gamma_{5}<1, \\
\rho_{12}{ }^{2}<\rho_{11}, \\
\rho_{13}{ }^{2}<\rho_{11} \rho_{33}, \\
\rho_{23}{ }^{2}<\rho_{33}, \\
(y-\bar{y})^{2}<\frac{1}{2}\left[(y-\bar{y})+\sqrt{\rho_{33}} z\right]^{2},
\end{gathered}
$$

where all the symbols are given in the proof.

Proof. Define $L_{3}(x, y, z)=\int_{\bar{x}}^{x}((m-x) / m) d m+\int_{\bar{y}}^{y}((w-y) / w)$ $d w+z$. Clearly, the function $L_{3}(x, y, z)$ is a positive definite function that is $L_{3}(\bar{x}, \bar{y}, 0)=0$, while $L_{3}(x, y, z)>0$, for all values in the region $\left\{(x, y, z) \in \mathbb{R}_{+}^{3}: x>0, y>0, z \geq 0 ;(x, y\right.$, $z) \neq(\bar{x}, \bar{y}, 0)\}$. Then, using some algebraic manipulation gives that

$$
\begin{aligned}
\frac{d L_{3}}{d t}= & (x-\bar{x})\left[-\frac{\alpha_{1}(y-\bar{y})}{\Gamma_{3} \bar{\Gamma}_{3}}-\frac{\alpha_{2} z}{\Gamma_{3} \bar{\Gamma}_{3}}-(x-\bar{x})-\frac{(y-\bar{y})}{\Gamma_{2}}\right. \\
& \left.+\frac{\alpha_{3} \bar{y}}{\Gamma_{2} \bar{\Gamma}_{2}}(x+\bar{x})(x-\bar{x})-\frac{z}{\Gamma_{1}}\right] \\
& +(y-\bar{y})\left[\frac{\alpha_{6}}{\Gamma_{2} \bar{\Gamma}_{2} \Gamma_{4}}\left(1-\alpha_{3} \bar{x} x\right)(x-\bar{x})-\frac{\alpha_{6} \alpha_{7} \bar{x} z}{\bar{\Gamma}_{2} \Gamma_{4}}-\frac{\alpha_{8} z}{\Gamma_{1}}\right] \\
& +\alpha_{10} z^{2}\left[1-\frac{1}{\Gamma_{5}}\right] .
\end{aligned}
$$

Furthermore, computation gives

$$
\begin{aligned}
\frac{d L_{3}}{d t}= & -\left[1-\frac{\alpha_{3} \bar{y}}{\Gamma_{2} \bar{\Gamma}_{2}}(x+\bar{x})\right](x-\bar{x})^{2} \\
& -\left[\frac{\alpha_{1}}{\Gamma_{3} \bar{\Gamma}_{3}}+\frac{1}{\Gamma_{2}}-\frac{\alpha_{6}\left(1-\alpha_{3} \bar{x} x\right)}{\Gamma_{2} \bar{\Gamma}_{2} \Gamma_{4}}\right](x-\bar{x})(y-\bar{y}) \\
& -\left[\frac{\alpha_{2}}{\Gamma_{3} \bar{\Gamma}_{3}}+\frac{1}{\Gamma_{1}}\right](x-\bar{x}) z-\left[\frac{\alpha_{6} \alpha_{7} \bar{x}}{\bar{\Gamma}_{2} \Gamma_{4}}+\frac{\alpha_{8}}{\Gamma_{1}}\right](y-\bar{y}) z \\
& -\alpha_{10} z^{2}\left[\frac{1}{\Gamma_{5}}-1\right] .
\end{aligned}
$$

Therefore, using conditions (59a), (59b), (59c), and (59d) gives that

$$
\begin{aligned}
\frac{d L_{3}}{d t} \leq & -\frac{1}{2}\left[\sqrt{\rho_{11}}(x-\bar{x})+(y-\bar{y})\right]^{2}-\frac{1}{2}\left[\sqrt{\rho_{11}}(x-\bar{x})\right. \\
& \left.+\sqrt{\rho_{33}} z\right]^{2}-\frac{1}{2}\left[(y-\bar{y})+\sqrt{\rho_{33}} z\right]^{2}+(y-\bar{y})^{2},
\end{aligned}
$$

where $\rho_{11}=1-\left(\alpha_{3} \bar{y} / \Gamma_{2} \bar{\Gamma}_{2}\right)(x+\bar{x}), \rho_{12}=\left(\alpha_{1} / \Gamma_{3} \bar{\Gamma}_{3}\right)+\left(1 / \Gamma_{2}\right)$ $-\left(\alpha_{6}\left(1-\alpha_{3} \bar{x} x\right) / \Gamma_{2} \bar{\Gamma}_{2} \Gamma_{4}\right), \quad \rho_{13}=\left(\alpha_{2} / \Gamma_{3} \bar{\Gamma}_{3}\right)+\left(1 / \Gamma_{1}\right), \quad \rho_{23}=($ $\left.\alpha_{6} \alpha_{7} \bar{x} / \bar{\Gamma}_{2} \Gamma_{4}\right)+\left(\alpha_{8} / \Gamma_{1}\right), \rho_{33}=\alpha_{10}\left[\left(1 / \Gamma_{5}\right)-1\right]$.

Accordingly, using condition (59f), it is observed that $\left(d L_{3} / d t\right)<0$, which means that $L_{3}$ is a Lyapunov function and hence the GPFP is an A.S for any trajectory starting from a point that belongs to the region that satisfies the above conditions.

Theorem 7. Assume that the SPFP is L.A.S; then, it has a basin of attraction that belongs to the $\mathbb{R}_{+}^{3}$ that satisfies the following conditions:

$$
\begin{gathered}
\frac{\alpha_{4} \overline{\bar{z}}(x+\bar{x})}{\Gamma_{1} \overline{\overline{\Gamma_{1}}}}<1, \\
F_{5}<1, \\
{q_{13}}^{2}<4 q_{11} q_{33}, \\
\frac{\alpha_{5} \overline{\bar{z}}}{\Gamma_{1} \overline{\overline{\Gamma_{1}}} y<\frac{\alpha_{1}}{\Gamma_{3} \overline{\overline{\Gamma_{3}}}},}
\end{gathered}
$$




$$
\begin{gathered}
\left(\frac{1}{\Gamma_{2}}+\frac{\alpha_{1}}{\Gamma_{3} \overline{\overline{\Gamma_{3}}}}\right) \overline{\bar{x}}<\alpha_{9} \\
\frac{\alpha_{10} \alpha_{13} \overline{\bar{z}}}{\Gamma_{5} \overline{\overline{\Gamma_{5}}}}<\frac{\alpha_{8}}{\Gamma_{1}}
\end{gathered}
$$

where the symbols $q_{11}, q_{33}$, and $q_{13}$ are given in the proof.

Proof. Define $L_{4}(x, y, z)=\int_{\overline{\bar{x}}}^{x}((m-\overline{\bar{x}}) / m) d m+y+\int_{\overline{\bar{z}}}^{z}((w-\overline{\bar{z}})$ $/ w) d w$. Clearly, the function $L_{4}(x, y, z)$ is a positive definite function that is $L_{4}(\overline{\bar{x}}, 0, \overline{\bar{z}})=0$, while $L_{4}(x, y, z)>0$, for all values in the region $\left\{(x, y, z) \in \mathbb{R}_{+}^{3}: x>0, y \geq 0, z>0 ;(x, y\right.$, $z) \neq(\overline{\bar{x}}, 0, \overline{\bar{z}})\}$. Then, using some algebraic manipulation gives that

$$
\begin{aligned}
& \frac{d L_{4}}{d t} \leq-\left[1-\frac{\alpha_{4} \overline{\bar{z}}(x+\overline{\bar{x}})}{\Gamma_{1} \overline{\overline{\Gamma_{1}}}}\right](x-\overline{\bar{x}})^{2}-\alpha_{10}\left[\frac{1}{\Gamma_{5}}-1\right](z-\overline{\bar{z}})^{2} \\
& -\left[\frac{\alpha_{2}}{\Gamma_{3} \overline{\bar{\Gamma}}_{3}}+\frac{1}{\Gamma_{1}}-\frac{\alpha_{10} \alpha_{12} \overline{\bar{z}}}{\Gamma_{5} \overline{\bar{\Gamma}}_{5}}\right](x-\overline{\bar{x}})(z-\overline{\bar{z}}) \\
& -\left[\alpha_{9}-\left(\frac{1}{\Gamma_{2}}+\frac{\alpha_{1}}{\Gamma_{3} \overline{\overline{\Gamma_{3}}}}\right) \overline{\bar{x}}\right] y-\left[1-\frac{\alpha_{6}}{\Gamma_{4}}\right] \frac{x y}{\Gamma_{2}} \\
& -\left[\frac{\alpha_{8}}{\Gamma_{1}}-\frac{\alpha_{10} \alpha_{13} \overline{\bar{z}}}{\Gamma_{5} \overline{\overline{\Gamma_{5}}}}\right] y z-\left[\frac{\alpha_{1}}{\Gamma_{3} \overline{\overline{\Gamma_{3}}}}-\frac{\alpha_{5} \overline{\bar{z}}}{\Gamma_{1} \overline{\overline{\Gamma_{1}}}} y\right] x y .
\end{aligned}
$$

Consequently, using conditions (63a), (63b), (63d), (63e), and (63f) gives

$$
\begin{aligned}
\frac{d L_{4}}{d t} \leq & -q_{11}(x-\overline{\bar{x}})^{2}-q_{33}(z-\overline{\bar{z}})^{2}-q_{13}(x-\overline{\bar{x}})(z-\overline{\bar{z}}) \\
- & {\left[\alpha_{9}-\left(\frac{1}{\Gamma_{2}}+\frac{\alpha_{1}}{\Gamma_{3} \overline{\overline{\Gamma_{3}}}}\right) \overline{\bar{x}}\right] y . }
\end{aligned}
$$

Moreover, using condition (63c) leads to

$$
\begin{gathered}
\frac{d L_{4} \leq}{d t} \leq-\left[\sqrt{q_{11}}(x-\overline{\bar{x}})+\sqrt{q_{33}}(z-\overline{\bar{z}})\right]^{2} \\
-\left[\alpha_{9}-\left(\frac{1}{\Gamma_{2}}+\frac{\alpha_{1}}{\Gamma_{3} \overline{\overline{\Gamma_{3}}}}\right) \overline{\bar{x}}\right] y .
\end{gathered}
$$

Accordingly, $\left(d L_{4} / d t\right)<0$, which means that $L_{4}$ is a Lyapunov function and hence the SPFP is A.S for any trajectory starting from a point that belongs to the region that satisfies the above conditions.

Theorem 8. Assume that the CP is L.A.S; then, it has a basin of attraction that belongs to the $\mathbb{R}_{+}{ }^{3}$ that satisfies the following conditions:

$$
d_{12}^{2}<d_{11} d_{22}
$$

$$
\begin{gathered}
d_{13}{ }^{2}<d_{11} d_{33}, \\
d_{23}{ }^{2}<d_{22} d_{33}, \\
\frac{\alpha_{3} y^{*}\left(x+x^{*}\right)}{\Gamma_{2} \Gamma_{2 *}}+\frac{\alpha_{4} z^{*}\left(x+x^{*}\right)}{\Gamma_{1} \Gamma_{1 *}}<1, \\
\alpha_{3} x x^{*}<1, \\
\alpha_{5} y^{* 2}<\alpha_{4} x^{* 2},
\end{gathered}
$$

where the symbols $d_{i j}, i, j=1,2,3$ are given in the proof.

Proof. Define

$$
L_{5}(x, y, z)=\int_{x^{*}}^{x} \frac{\left(m-x^{*}\right)}{m} d m+\int_{y^{*}}^{y} \frac{\left(w-y^{*}\right)}{w} d w+\int_{z^{*}}^{z} \frac{\left(n-z^{*}\right)}{n} d n
$$

Clearly, the function $L_{5}(x, y, z)$ is a positive definite function that is $L_{5}\left(x^{*}, y^{*}, z^{*}\right)=0$, while $L_{5}(x, y, z)>0$, for all values in the region $\left\{(x, y, z) \in \mathbb{R}_{+}^{3}: x>0, y>0, x>0\right.$; $\left.(x, y, z) \neq\left(x^{*}, y^{*}, z^{*}\right)\right\}$. Thus, after some algebraic manipulation, it is obtained that

$$
\begin{aligned}
\frac{d L_{5}}{d t}= & -\frac{d_{11}}{2}\left(x-x^{*}\right)^{2}-d_{12}\left(x-x^{*}\right)\left(y-y^{*}\right)-\frac{d_{22}}{2}\left(y-y^{*}\right)^{2} \\
& -\frac{d_{33}}{2}\left(z-z^{*}\right)^{2}-d_{13}\left(x-x^{*}\right)\left(z-z^{*}\right) \\
& -d_{23}\left(y-y^{*}\right)\left(z-z^{*}\right),
\end{aligned}
$$

where

$$
\begin{aligned}
d_{11}= & -\frac{\alpha_{3} y^{*}\left(x+x^{*}\right)}{\Gamma_{2} \Gamma_{2 *}}+\frac{\alpha_{4} z^{*}\left(x+x^{*}\right)}{\Gamma_{1} \Gamma_{1 *}}, \\
d_{12}=1 & -\frac{\alpha_{1}}{\Gamma_{3} \Gamma_{3 *}}+\frac{1+\alpha_{3} x^{*}}{\Gamma_{2} \Gamma_{2 *}} \\
& +\frac{\left(\alpha_{3}+\alpha_{7} z\right) \alpha_{6} x x^{*}-\alpha_{6}\left(1+\alpha_{7} z^{*}\right)}{\Gamma_{2} \Gamma_{2 *} \Gamma_{4} \Gamma_{4 *}} \\
& +\frac{\alpha_{5} z^{*}\left(y+y^{*}\right)-\alpha_{4} \alpha_{8}\left(x+x^{*}\right)}{\Gamma_{2} \Gamma_{2 *}}, \\
d_{22}= & \frac{\alpha_{5} \alpha_{8} z^{*}\left(y+y^{*}\right)}{\Gamma_{1} \Gamma_{1 *}}, \\
d_{33}= & \alpha_{10}+\frac{\alpha_{10}\left(\alpha_{11}+\alpha_{13} x^{*}\right)}{\Gamma_{5} \Gamma_{5 *}}+\frac{\alpha_{10} \alpha_{13} y^{*}}{\Gamma_{2} \Gamma_{2 *} \Gamma_{4} \Gamma_{4 *}}, \\
d_{13}= & \frac{\alpha_{2}}{\Gamma_{3} \Gamma_{3 *}}+\frac{1}{\Gamma_{1}}-\frac{\alpha_{10} \alpha_{12} z^{*}}{\Gamma_{5} \Gamma_{5 *}}, \\
d_{23}= & \frac{\alpha_{6} \alpha_{7} x^{*}\left(1-\alpha_{3} x^{*}\right)}{\Gamma_{2} \Gamma_{2 *} \Gamma_{4} \Gamma_{4 *}}+\frac{\alpha_{8}}{\Gamma_{1} \Gamma_{1 *}}+\frac{\alpha_{4} \alpha_{8} x^{* 2}}{\Gamma_{1} \Gamma_{1 *}} \\
& +\frac{\alpha_{8} \alpha_{5} y^{* 2}}{\Gamma_{1} \Gamma_{1 *}}-\frac{\alpha_{10} \alpha_{13} z^{*}}{\Gamma_{5} \Gamma_{5 *}} .
\end{aligned}
$$


Consequently, using the above conditions gives

$$
\begin{aligned}
\frac{d L_{5} \leq}{d t} \leq & -\frac{1}{2}\left[\sqrt{d_{11}}\left(x-x^{*}\right)+\sqrt{d_{22}}\left(y-y^{*}\right)\right]^{2} \\
& -\frac{1}{2}\left[\sqrt{d_{22}}\left(y-y^{*}\right)+\sqrt{d_{33}}\left(z-z^{*}\right)\right]^{2} \\
& -\frac{1}{2}\left[\sqrt{d_{11}}\left(x-x^{*}\right)+\sqrt{d_{33}}\left(z-z^{*}\right)\right]^{2} .
\end{aligned}
$$

Obviously, $\left(d L_{5} / d t\right)<0$, which gives that $L_{5}$ is a Lyapunov function and hence the CP is A.S for any trajectory starting from a point that belongs to the region that satisfies the above conditions.

\section{Local Bifurcation}

An investigation of the impact of varying the parameter values on the dynamics of system (4) around each equilibrium point is done using Sotomayor's theorem for local bifurcation. Because the existence of a nonhyperbolic equilibrium point represents a necessary but not sufficient condition for bifurcation to occur, then the candidate bifurcation parameter is choosing so that the equilibrium point becomes a nonhyperbolic point. Rewrite system (4) in the following general form:

$$
\frac{d X}{d t}=F(X), X=(x, y, z)^{T}, F=\left(x f_{1}, y f_{2}, z f_{3}\right)^{T}
$$

Then, the second directional derivative of system (4) can become as

$$
D^{2} F(X)(\Phi, \Phi)=\left[\varphi_{\mathrm{i} 1}\right]_{3 \times 1}
$$

where

$$
\begin{aligned}
\varphi_{11}= & \left(-2+\frac{2 \alpha_{3} x y\left(3-\alpha_{3} x^{2}\right)}{\Gamma_{2}^{3}}+\frac{2 \alpha_{4} x z\left(3-\alpha_{4} x^{2}+3 \alpha_{5} y^{2}\right)}{\Gamma_{1}^{3}}\right) \\
& \cdot v_{1}{ }^{2}+\left(-\frac{2 \alpha_{1}}{\Gamma_{3}^{2}}-\frac{2\left(1-\alpha_{3} x^{2}\right)}{\Gamma_{2}^{2}}+\frac{\alpha_{5} y z\left(4+4 \alpha_{5} y^{2}-10 \alpha_{4} x^{2}\right)}{\Gamma_{1}^{3}}\right) \\
& \cdot v_{1} v_{2}-\left(\frac{2 \alpha_{2}}{\Gamma_{3}^{2}}+\frac{2\left(1-\alpha_{4} x^{2}+\alpha_{5} y^{2}\right)}{\Gamma_{1}}\right) \\
& \cdot v_{1} v_{3}+\left(\frac{2 \alpha_{1}^{2} x}{\Gamma_{3}^{3}}+\frac{2 \alpha_{5} x z\left(1+\alpha_{4} x^{2}-2 \alpha_{5} y^{2}\right)}{\Gamma_{1}^{3}}\right) \\
& \cdot v_{2}{ }^{2}+4 x\left(\frac{\alpha_{1} \alpha_{2}}{\Gamma_{3}^{3}}+\frac{\alpha_{5} y}{\Gamma_{1}^{2}}\right) v_{2} v_{3}+\left(\frac{2 \alpha_{2}{ }^{2} x}{\Gamma_{3}^{3}}\right) v_{3}{ }^{2}, \\
\varphi_{21}= & \left.\frac{2 \alpha_{3} \alpha_{6} x y\left(-3+\alpha_{3} x^{2}\right)}{\Gamma_{2}^{3} \Gamma_{4}}+\frac{2 \alpha_{4} \alpha_{8} y z\left(1+\alpha_{5} y^{2}-2 \alpha_{4} x^{2}\right)}{\Gamma_{1}^{3}}\right) \\
& \cdot v_{1}{ }^{2}+\left(\frac{\alpha_{4} \alpha_{8} x z\left(4+4 \alpha_{4} x^{2}-10 \alpha_{5} y^{2}\right)}{\Gamma_{1}^{3}}+\frac{2 \alpha_{6}\left(1-\alpha_{3} x^{2}\right)}{\Gamma_{2}^{2} \Gamma_{4}}\right) \\
& \cdot v_{1} v_{2}+\left(-\frac{2 \alpha_{6} \alpha_{7} y\left(1-\alpha_{3} x^{2}\right)}{\Gamma_{2}^{2} \Gamma_{4}^{2}}+\frac{4 \alpha_{4} \alpha_{8} x y}{\Gamma_{1}^{2}}\right) v_{1} v_{3} \\
& +\left(\frac{2 \alpha_{5} \alpha_{8} y z\left(3-\alpha_{5} y^{2}+3 \alpha_{4} x^{2}\right)}{\Gamma_{1}^{3}}\right) v_{2}{ }^{2} \\
& +\left(\frac{\alpha_{6} \alpha_{7}{ }^{2} x y}{\Gamma_{2} \Gamma_{4}^{3}}\right) v_{3}{ }^{2}-\left(\frac{2 \alpha_{6} \alpha_{7} x}{\Gamma_{2} \Gamma_{4}^{2}}+\frac{2 \alpha_{8}\left(1+\alpha_{4} x^{2}-\alpha_{5} y z\right)}{\Gamma_{1}^{2}}\right) v_{2} v_{3}, \\
\varphi_{31}= & \left(-\frac{\alpha_{10} \alpha_{12}{ }^{2} z^{2}}{\Gamma_{5}^{3}}\right) v_{1}{ }^{2}-\left(2 \frac{\alpha_{10} \alpha_{12} \alpha_{13} z^{2}}{\Gamma_{5}^{3}}\right) v_{1} v_{2} \\
& +4 z\left(\frac{\alpha_{10} \alpha_{12}}{\Gamma_{5}^{2}}\right) v_{1} v_{3}-\left(\frac{\alpha_{10} \alpha_{13}{ }^{2} z^{2}}{\Gamma_{5}^{3}}\right) v_{2}{ }^{2} \\
& +4 z\left(\frac{\alpha_{10} \alpha_{13}}{\Gamma_{5}^{2}}\right) v_{2} v_{3}+2\left(\alpha_{10}-\frac{\alpha_{10}}{\Gamma_{5}}\right) v_{3}^{2},
\end{aligned}
$$

with $\Phi=\left(v_{1}, v_{2}, v_{3}\right)^{T}$ be any nonzero vector, and the symbols $\Gamma_{i} ; i=1,2,3,4,5$ are given in equation (20). Accordingly, the local bifurcation near the equilibrium points is studied in the next theorems.

Theorem 9. Suppose that condition (32a) is satisfied; then, system (4) at the SPFP undergoes a transcritical bifurcation when the parameter $\alpha_{9}$ passes through the value $\alpha_{9}^{*}=\left(\alpha_{6} \overline{\bar{x}} /\right.$ $\left.\overline{\bar{\Gamma}}_{2} \overline{\bar{\Gamma}}_{4}\right)-\left(\alpha_{8} \overline{\bar{z}} / \overline{\bar{\Gamma}}_{1}\right)$, provided that the following condition holds:

$$
\begin{gathered}
\left(\frac{4 \alpha_{4} \alpha_{8} \overline{\bar{x}} \overline{\bar{z}}\left(1+\alpha_{4} \overline{\bar{x}}^{2}\right)}{\overline{\bar{\Gamma}}_{1}^{3}}+\frac{2 \alpha_{6}\left(1-\alpha_{3} \overline{\bar{x}}^{2}\right)}{\overline{\bar{\Gamma}}_{2}^{2} \overline{\bar{\Gamma}}_{4}}\right) \\
\cdot \rho_{1}-\left(\frac{2 \alpha_{6} \alpha_{7} \overline{\bar{x}}}{\overline{\bar{\Gamma}}_{2} \overline{\bar{\Gamma}}_{4}^{2}}+\frac{2 \alpha_{8}\left(1+\alpha_{4} \overline{\bar{x}}^{2}\right)}{\overline{\bar{\Gamma}}_{1}^{2}}\right) \rho_{2} \neq 0,
\end{gathered}
$$

where the symbols $\rho_{1}$ and $\rho_{2}$ are given in the proof.

Proof. Direct computation shows that the JM of system (4) at $\left(E_{3}, \alpha_{9}^{*}\right)$ can be represented as

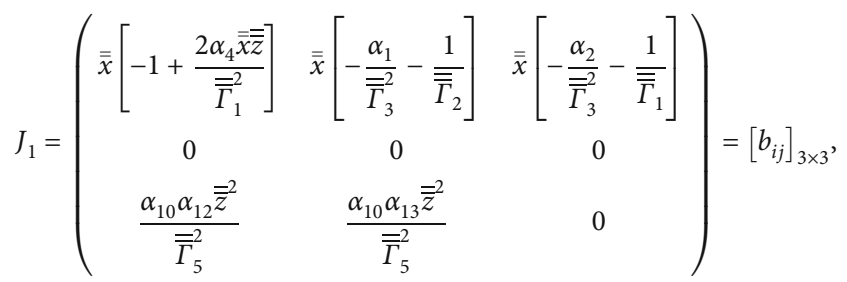

where $\overline{\bar{\Gamma}}_{i} ; i=1,2,3,4,5$ are given in equation (28). Obviously, the matrix $J_{1}$ has two eigenvalues having negative real parts due to condition (32a) and the third one is zero, say $\lambda_{32}{ }^{*}=0$. Hence, $E_{3}$ is a nonhyperbolic point.

Let $\Theta_{1}=\left(\theta_{11}, \theta_{12}, \theta_{13}\right)^{T}$ be the eigenvector corresponding to the eigenvalue $\lambda_{32}{ }^{*}=0$. Thus, $J_{1} \Theta_{1}=0$ gives that $\Theta_{1}=\left(-\left(b_{32} / b_{31}\right) \theta_{12}, \theta_{12},\left(b_{11} b_{32}-b_{31} b_{12} / b_{31} b_{13}\right) \theta_{12}\right)^{T}$ $=\left(\rho_{1} \theta_{12}, \theta_{12}, \rho_{2} \theta_{12}\right)^{T}$, where $\theta_{12} \neq 0$ be any real number.

Now, let $Y_{1}=\left(\epsilon_{11}, \epsilon_{12}, \epsilon_{13}\right)^{T}$ represent the eigenvector corresponding to the eigenvalue $\lambda_{32}{ }^{*}=0$ of the matrix $J_{1}{ }^{T}$. Thus, $J_{1}{ }^{T} Y_{1}=0$ gives that $Y_{1}=\left(0, \epsilon_{12}, 0\right)^{T}$, where $\epsilon_{12} \neq 0$ be any real number. Now, according to Sotomayor's theorem, it is obtained that

$$
\frac{\partial F}{\partial \alpha_{9}}=F_{\alpha_{9}}\left(X, \alpha_{9}\right)=(0,-y, 0)^{T} \Rightarrow \frac{\partial F}{\partial \alpha_{9}}=F_{\alpha_{9}}\left(E_{3}, \alpha_{9}^{*}\right)=(0,0,0)^{T} .
$$

Therefore, $Y_{1}{ }^{T} F_{\alpha_{9}}\left(E_{3}, \alpha_{9}^{*}\right)=0$; hence, system (4) has no 
saddle-node bifurcation. Moreover, since

$D F_{\alpha_{9}}\left(X, \alpha_{9}\right)=\left(\begin{array}{ccc}0 & 0 & 0 \\ 0 & -1 & 0 \\ 0 & 0 & 0\end{array}\right) \Rightarrow D F_{\alpha_{9}}\left(E_{3}, \alpha_{9}^{*}\right) \Theta_{1}=\left(0,-\theta_{12}, 0\right)^{T}$

Then, $Y_{1}^{T} D F_{\alpha_{9}}\left(E_{3}, \alpha_{9}^{*}\right) \Theta_{1}=-\theta_{12} \epsilon_{12} \neq 0$.

Also, by using equation (73), it is obtained that

$$
D^{2} F\left(E_{3}, \alpha_{9}^{*}\right)\left(\Theta_{1}, \Theta_{1}\right)=\left[\overline{\bar{\varphi}}_{i 1}\right]_{3 \times 1},
$$

where

$$
\begin{aligned}
& \overline{\bar{\varphi}}_{11}=\left(-2+\frac{2 \alpha_{4} \overline{\bar{x}}\left(3-\alpha_{4} \overline{\bar{x}}^{2}\right)}{\overline{\bar{\Gamma}}_{1}^{3}}\right)\left(\rho_{1} \theta_{12}\right)^{2}-\left(\frac{2 \alpha_{1}}{\overline{\bar{\Gamma}}_{3}^{2}}+\frac{2\left(1-\alpha_{3} \overline{\bar{x}}^{2}\right)}{\overline{\bar{\Gamma}}_{2}^{2}}\right) \rho_{1} \theta_{12}{ }^{2} \\
& +4 \overline{\bar{x}}\left(\frac{\alpha_{1} \alpha_{2}}{\overline{\bar{\Gamma}}_{3}^{3}}\right) \rho_{2} \theta_{12}^{2}-\left(\frac{2 \alpha_{2}}{\overline{\bar{\Gamma}}_{3}^{2}}+\frac{2\left(1-\alpha_{4} \overline{\bar{x}}^{2}\right)}{\overline{\bar{\Gamma}}_{1}}\right) \rho_{1} \rho_{2} \theta_{12}^{2} \\
& +\left(\frac{2 \alpha_{1}^{2} \overline{\bar{x}}}{\overline{\bar{\Gamma}}_{3}^{3}}+\frac{2 \alpha_{5} \overline{\bar{x}} \overline{\bar{z}}\left(1+\alpha_{4} \overline{\bar{x}}^{2}\right)}{\overline{\bar{\Gamma}}_{1}^{3}}\right) \theta_{12}{ }^{2}+\left(\frac{2 \alpha_{2}^{2} \overline{\bar{x}}}{\overline{\bar{\Gamma}}_{3}^{3}}\right)\left(\rho_{2} \theta_{12}\right)^{2}, \\
& \overline{\bar{\varphi}}_{21}=\left(\frac{4 \alpha_{4} \alpha_{8} \overline{\bar{x}}\left(1+\alpha_{4} \overline{\bar{x}}^{2}\right)}{\overline{\bar{\Gamma}}_{1}^{3}}+\frac{2 \alpha_{6}\left(1-\alpha_{3} \overline{\bar{x}}^{2}\right)}{\overline{\bar{\Gamma}}_{2}^{2} \overline{\bar{\Gamma}}_{4}}\right) \rho_{1} \theta_{12}^{2} \\
& -\left(\frac{2 \alpha_{6} \alpha_{7} \overline{\bar{x}}}{\overline{\bar{\Gamma}}_{2} \overline{\bar{\Gamma}}_{4}^{2}}+\frac{2 \alpha_{8}\left(1+\alpha_{4} \overline{\bar{x}}^{2}\right)}{\overline{\bar{\Gamma}}_{1}^{2}}\right) \rho_{2} \theta_{12}^{2} \\
& \overline{\bar{\varphi}}_{31}=\left(-\frac{\alpha_{10} \alpha_{12}{ }^{2} \overline{\bar{z}}^{2}}{\overline{\bar{\Gamma}}_{5}^{3}}\right)\left(\rho_{1} \theta_{12}\right)^{2}-\left(2 \frac{\alpha_{10} \alpha_{12} \alpha_{13} \overline{\bar{z}}^{2}}{\overline{\bar{\Gamma}}_{5}^{3}}\right) \rho_{1} \theta_{12}{ }^{2} \\
& +4 \overline{\bar{z}}\left(\frac{\alpha_{10} \alpha_{12}}{\overline{\bar{\Gamma}}_{5}^{2}}\right) \rho_{1} \rho_{2} \theta_{12}^{2}-\left(\frac{\alpha_{10} \alpha_{13}^{2} \overline{\bar{z}}^{2}}{\overline{\bar{\Gamma}}_{5}^{3}}\right) \theta_{12}^{2} \\
& +4 \overline{\bar{z}}\left(\frac{\alpha_{10} \alpha_{13}}{\overline{\bar{\Gamma}}_{5}^{2}}\right) \rho_{2} \theta_{12}{ }^{2}+2\left(\alpha_{10}-\frac{\alpha_{10}}{\overline{\bar{\Gamma}}_{5}}\right)\left(\rho_{2} \theta_{12}\right)^{2} \text {. }
\end{aligned}
$$

Accordingly, it is easy to verify that

$$
\begin{aligned}
Y_{1}{ }^{T} D^{2} F\left(E_{3}, \alpha_{9}^{*}\right)\left(\Theta_{1}, \Theta_{1}\right) \\
=\left[\left(\frac{4 \alpha_{4} \alpha_{8} \overline{\bar{x} \bar{z}}\left(1+\alpha_{4} \overline{\bar{x}}^{2}\right)}{\overline{\bar{\Gamma}}_{1}^{3}}+\frac{2 \alpha_{6}\left(1-\alpha_{3} \overline{\bar{x}}^{2}\right)}{\left.\overline{\bar{\Gamma}}_{2}^{2} \overline{\bar{\Gamma}}_{4}\right)}\right.\right. \\
\left.\cdot \rho_{1}-\left(\frac{2 \alpha_{6} \alpha_{7} \overline{\bar{x}}}{\overline{\bar{\Gamma}}_{2} \overline{\bar{\Gamma}}_{4}^{2}}+\frac{2 \alpha_{8}\left(1+\alpha_{4} \overline{\bar{x}}^{2}\right)}{\overline{\bar{\Gamma}}_{1}^{2}}\right) \rho_{2}\right] \theta_{12}^{2} \epsilon_{12} .
\end{aligned}
$$

Clearly, $Y_{1}^{\mathrm{T}} D^{2} F\left(E_{3}, \alpha_{9}^{*}\right)\left(\Theta_{1}, \Theta_{1}\right) \neq 0$, under condition (75), and then, a transcritical bifurcation take place in the sense of Sotomayor.

Theorem 10. Suppose that conditions (38a)-(38c) and (38e) are satisfied; then, system (4) at CP undergoes a saddle- node bifurcation when the parameter $\alpha_{12}$ passes through the value $\alpha_{12}^{*}=\left(\alpha_{13}\left(c_{11} c_{23}-c_{13} c_{21}\right)\right) /\left(c_{12} c_{23}-c_{13} c_{22}\right)$, provided that the following condition holds:

$$
\rho_{5} \varphi_{11}^{*}+\rho_{6} \varphi_{21}^{*}+\varphi_{31}^{*} \neq 0
$$

where $\varphi_{i 1}^{*} ; i=1,2,3$ are given in the proof.

Proof. Direct computation shows that the JM of system (4) at $\left(E_{4}, \alpha_{12}^{*}\right)$ can be represented as

$$
J_{2}=\left(\begin{array}{ccc}
c_{11} & c_{12} & c_{13} \\
c_{21} & c_{22} & c_{23} \\
c_{31}\left(\alpha_{12}^{*}\right) & c_{32}\left(\alpha_{12}^{*}\right) & 0
\end{array}\right)
$$

where all the coefficients $c_{i j} ; i, j=1,2,3$ are given in equation (33). Clearly, the determinant of $J_{2}$ that is given by $A_{3}$ in equation (35) is zero at $\alpha_{12}^{*}$. Therefore, the characteristic equation of $J_{2}$ has zero root, say $\lambda^{*}=0$, and then, the coexistence equilibrium point becomes a nonhyperbolic point.

Let $\Theta_{2}=\left(\theta_{21}, \theta_{22}, \theta_{23}\right)^{T}$ be the eigenvector corresponding to the eigenvalue $\lambda^{*}=0$. Thus, $J_{2} \Theta_{2}=0$ gives that $\Theta_{2}$ $=\left(\rho_{4} \theta_{23}, \rho_{3} \theta_{23}, \theta_{23}\right)^{T}$, where $\theta_{23} \neq 0$ be any real number, and $\rho_{3}=\left(c_{13} c_{21}-c_{11} c_{23}\right) /\left(c_{11} c_{22}-c_{12} c_{21}\right)$, while $\rho_{4}=\left(c_{23} c_{12}\right.$ $\left.-c_{13} c_{22}\right) /\left(c_{11} c_{22}-c_{12} c_{21}\right)$. Clearly, $\rho_{3}>0$, and $\rho_{4}<0$, due to conditions (38a) $-(38 \mathrm{c})$ with $(38 \mathrm{e})$.

Now, let $Y_{2}=\left(\epsilon_{21}, \epsilon_{22}, \epsilon_{23}\right)^{T}$ represent the eigenvector corresponding to the eigenvalue $\lambda^{*}=0$ of the matrix $J_{2}^{T}$. Thus, $J_{2}^{T} Y_{2}=0$ gives that $Y_{2}=\left(\rho_{5} \epsilon_{23}, \rho_{6} \epsilon_{23}, \epsilon_{23}\right)^{T}$, where $\epsilon_{23} \neq 0$ be any real number, and $\rho_{5}=-c_{23} c_{31} /\left(c_{11} c_{23}-c_{13}\right.$ $\left.c_{21}\right)$, while $\rho_{6}=c_{13} c_{31} /\left(c_{11} c_{23}-c_{13} c_{21}\right)$. Clearly, $\rho_{5}>0$, and $\rho_{6}<0$, due to conditions (38a) $-(38 \mathrm{c})$ with (38e).

Now, according to Sotomayor's theorem, it is obtained that

$$
\frac{\partial F}{\partial \alpha_{12}}=F_{\alpha_{12}}\left(X, \alpha_{12}\right)=\left(0,0, \frac{\alpha_{10} x z^{2}}{\Gamma_{5}^{2}}\right)^{T}
$$

where $\Gamma_{5}$ is given in equation (20). Therefore, it is obtained that

$$
Y_{2}^{*} F_{\alpha_{12}}\left(E_{4}, \alpha_{12}^{*}\right)=\frac{\alpha_{10} x^{*} z^{* 2}}{\Gamma_{5 *}^{2}} \epsilon_{23} \neq 0,
$$

where $\Gamma_{5 *}$ is given in equation (33). Now, by using equation (73), it is obtained that

$$
D^{2} F\left(E_{4}, \alpha_{12}^{*}\right)\left(\Theta_{2}, \Theta_{2}\right)=\left[\varphi_{i 1}^{*}\right]_{3 \times 1},
$$




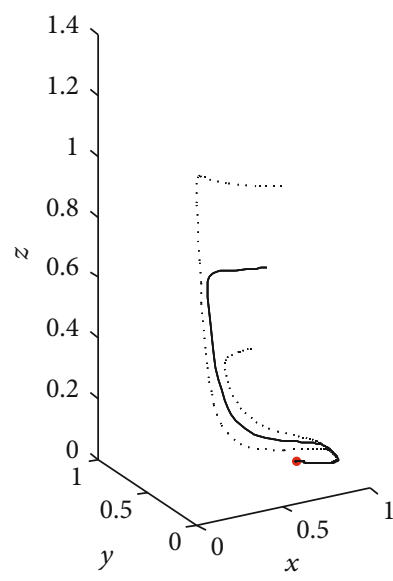

(a)
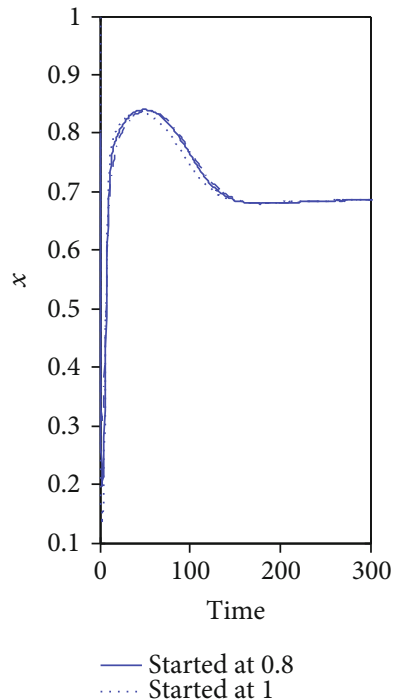

- - Started at 0.6

(b)

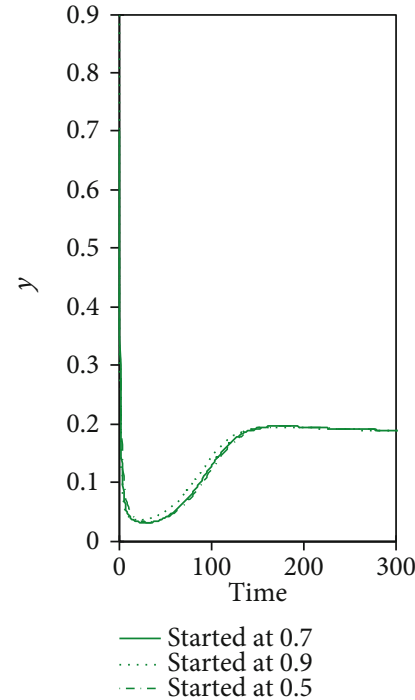

(c)

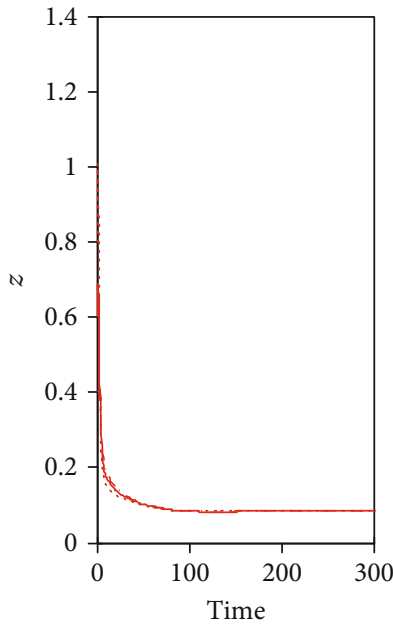

- Started at 0.6 -. Started at 0.4

(d)

FIGURE 1: (a) The trajectories of system (4) approach asymptotically to CP, starting from different initial points with the data given by (89).

(b) The trajectory of $x$ versus $t$. (c) The trajectory of $y$ versus $t$. (d) The trajectory of $z$ versus $t$.

where

$$
\begin{aligned}
\varphi_{11}^{*}= & -\left(2-\frac{2 \alpha_{3} x^{*} y^{*}\left(3-\alpha_{3} x^{* 2}\right)}{\Gamma_{2 *}^{3}}-\frac{2 \alpha_{4} x^{*} z^{*}\left(3-\alpha_{4} x^{* 2}+3 \alpha_{5} y^{* 2}\right)}{\Gamma_{1 *}^{3}}\right)\left(\rho_{4} \theta_{23}\right)^{2} \\
& -\left(\frac{2 \alpha_{1}}{\Gamma_{3}^{2}}+\frac{2\left(1-\alpha_{3} x^{* 2}\right)}{\Gamma_{2 *}^{2}}-\frac{\alpha_{5} y^{*} z^{*}\left(4+4 \alpha_{5} y^{* 2}-10 \alpha_{4} x^{* 2}\right)}{\Gamma_{1 *}^{3}}\right) \rho_{3} \rho_{4} \theta_{23}{ }^{2} \\
& -\left(\frac{2 \alpha_{2}}{\Gamma_{3 *}^{2}}+\frac{2\left(1-\alpha_{4} x^{* 2}+\alpha_{5} y^{* 2}\right)}{\Gamma_{1 *}}\right) \rho_{4} \theta_{23}^{2}+4 x^{*}\left(\frac{\alpha_{1} \alpha_{2}}{\Gamma_{3 *}^{3}}+\frac{\alpha_{5} y^{*}}{\Gamma_{1 *}^{2}}\right) \rho_{3} \theta_{23}^{2} \\
& +\left(\frac{2 \alpha_{1}{ }^{2} x^{*}}{\Gamma_{3}^{3}}+\frac{2 \alpha_{5} x^{*} z^{*}\left(1+\alpha_{4} x^{* 2}-2 \alpha_{5} y^{* 2}\right)}{\Gamma_{1 *}^{3}}\right)\left(\rho_{3} \theta_{23}\right)^{2}+\left(\frac{2 \alpha_{2}{ }^{2} x^{*}}{\Gamma_{3 *}^{3}}\right) \theta_{23}^{2}, \\
\varphi_{21}^{*}= & \left(\frac{2 \alpha_{3} \alpha_{6} x^{*} y^{*}\left(-3+\alpha_{3} x^{* 2}\right)}{\Gamma_{2 *}^{3} \Gamma_{4 *}}+\frac{2 \alpha_{4} \alpha_{8} y^{*} z^{*}\left(1+\alpha_{5} y^{* 2}-2 \alpha_{4} x^{* 2}\right)}{\Gamma_{1 *}^{3}}\right)\left(\rho_{4} \theta_{23}\right)^{2} \\
& +\left(\frac{\alpha_{4} \alpha_{8} x^{*} z^{*}\left(4+4 \alpha_{4} x^{* 2}-10 \alpha_{5} y^{* 2}\right)}{\Gamma_{1 *}{ }^{2}}+\frac{2 \alpha_{6}\left(1-\alpha_{3} x^{* 2}\right)}{\Gamma_{2 *}^{2} \Gamma_{4 *}}\right) \rho_{3} \rho_{4} \theta_{23}^{2} \\
& -\left(\frac{2 \alpha_{6} \alpha_{7} y^{*}\left(1-\alpha_{3} x^{* 2}\right)}{\Gamma_{2 *}^{2} \Gamma_{4 *}^{2}}-\frac{4 \alpha_{4} \alpha_{8} x^{*} y^{*}}{\Gamma_{1 *}^{2}}\right) \rho_{4} \theta_{23}^{2}+\left(\frac{\alpha_{6} \alpha_{7}{ }^{2} x^{*} y^{*}}{\Gamma_{2 *} \Gamma_{4 *}^{3}}\right) \theta_{23}^{2} \\
& +\left(\frac{2 \alpha_{5} \alpha_{8} y^{*} z^{*}\left(3-\alpha_{5} y^{* 2}+3 \alpha_{4} x^{* 2}\right)}{\Gamma_{1 *}^{3}}\right)\left(\rho_{3} \theta_{23}\right)^{2} \\
& -\left(\frac{2 \alpha_{6} \alpha_{7} x^{*}}{\Gamma_{2 *} \Gamma_{4 *}^{2}}+\frac{2 \alpha_{8}\left(1+\alpha_{4} x^{* 2}-\alpha_{5} y^{*} z^{*}\right)}{\Gamma_{1 *}^{2}}\right) \rho_{3} \theta_{23}^{2}, \\
\varphi_{31}^{*}= & -\left(\frac{\alpha_{10} \alpha_{12}^{*} z^{* 2}}{\Gamma_{5 *}^{3}}\right)\left(\rho_{4} \theta_{23}\right)^{2}-\left(2 \frac{\alpha_{10} \alpha_{12}^{*} \alpha_{13} z^{* 2}}{\Gamma_{5 *}^{3}}\right) \rho_{3} \rho_{4} \theta_{23}^{2} \\
& +4 z^{*}\left(\frac{\alpha_{10} \alpha_{12}^{*}}{\Gamma_{5 *}^{2}}\right) \rho_{4} \theta_{23}^{2}-\left(\frac{\alpha_{10} \alpha_{13}{ }^{2} z^{2}}{\Gamma_{5 *}^{3}}\right)\left(\rho_{3} \theta_{23}\right)^{2} \\
& +4 z^{*}\left(\frac{\alpha_{10} \alpha_{13}}{\Gamma_{5 *}^{2}}\right) \rho_{4} \theta_{23}^{2}+2\left(\alpha_{10}-\frac{\alpha_{10}}{\Gamma_{5 *}}\right) \theta_{23}^{2} .
\end{aligned}
$$

Accordingly, it is simple to verify that

$$
Y_{2}{ }^{T} D^{2} F\left(E_{4}, \alpha_{12}^{*}\right)\left(\Theta_{2}, \Theta_{2}\right)=\epsilon_{23}\left(\rho_{5} \varphi_{11}^{*}+\rho_{6} \varphi_{21}^{*}+\varphi_{31}^{*}\right) .
$$

Clearly, $Y_{2}{ }^{T} D^{2} F\left(E_{4}, \alpha_{12}^{*}\right)\left(\Theta_{2}, \Theta_{2}\right) \neq 0$, under condition (82), and then, a saddle-node bifurcation takes place in the sense of Sotomayor.

\section{Numerical Simulation}

In this part, the dynamics of system (4) are studied numerically. The objective is to complete the vision of the global dynamics of the system and detect the effect of varying the parameter values on the system's dynamical behavior. Two sets of hypothetical parameter values are used to satisfy our objective. For the following set of parameter values, other sets can be used to study the system:

$$
\begin{gathered}
\alpha_{1}=0.2, \alpha_{2}=0.2, \alpha_{3}=0.1, \alpha_{4}=0.1, \alpha_{5}=0.1, \alpha_{6}=0.25 \\
\alpha_{7}=0.2, \alpha_{8}=0.75, \alpha_{9}=0.1, \alpha_{10}=0.75, \alpha_{11}=0.1, \alpha_{12}=0.9, \alpha_{13}=1.5 .
\end{gathered}
$$

It is watched that system (4) has a globally asymptotically stable CP as shown in Figure 1.

According to Figure 1, system (4) is globally asymptotically stable given by $E_{4}=(0.68,0.18,0.08)$ which means system (4) is persistent at the CP. However, for the data (89) with increasing $\alpha_{1}$, system (4) loses its persistence and approaches asymptotically to GPFP as shown in Figure 2 for the typical value of $\alpha_{1}$.

On the other hand, varying the other fear's parameters leads to a quantitative change in the position of the $\mathrm{CP}$ and system (4) still persistent. Now, the effects of varying the parameters $\alpha_{3}$ and $\alpha_{5}$ are presented in Figures 3 and 4, respectively. 


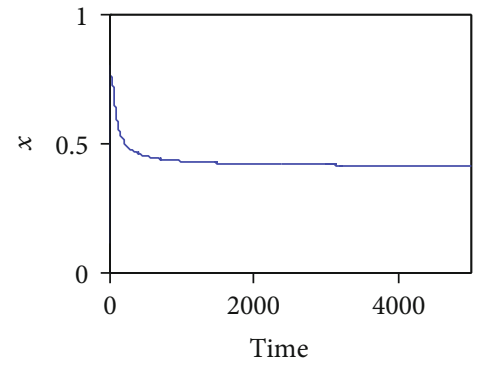

(a)

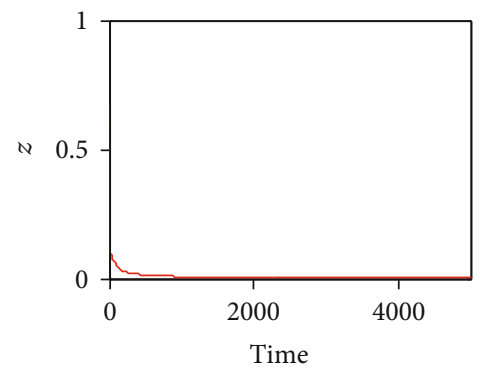

(c)

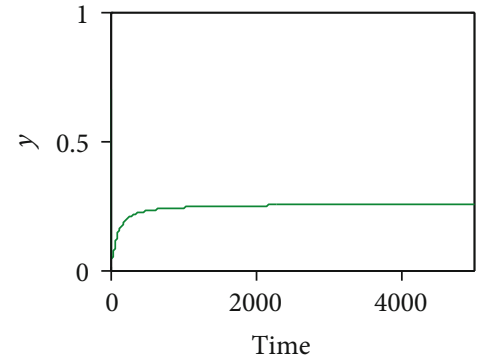

(b)

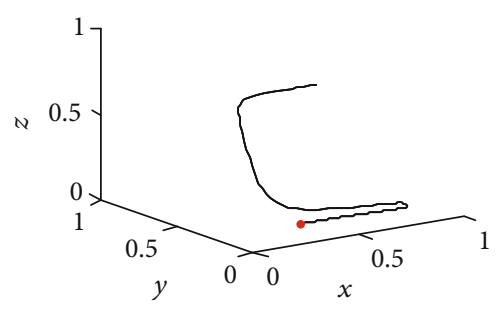

(d)

FIGURE 2: For $\alpha_{1}=2$, with the data given by (89). (a) The trajectory of $x$ versus $t$. (b) The trajectory of $y$ versus $t$. (c) The trajectory of $z$ versus t. (d) The trajectory of system (4) approaches asymptotically to GPFP.

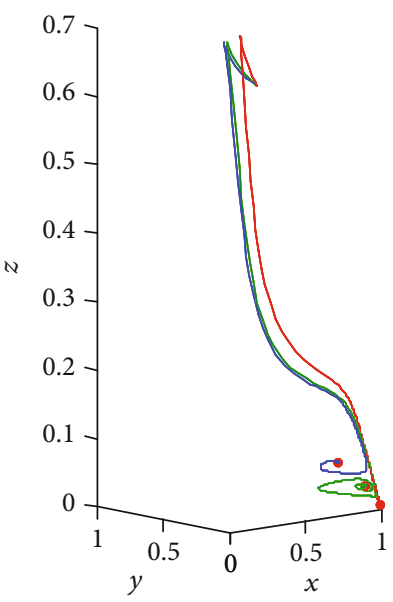

(a)

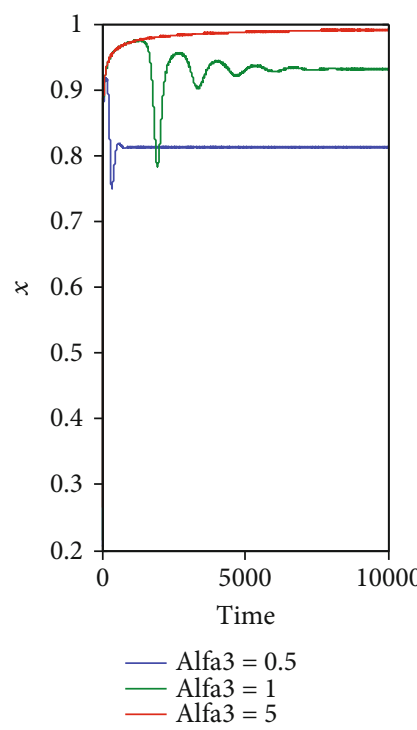

(b)
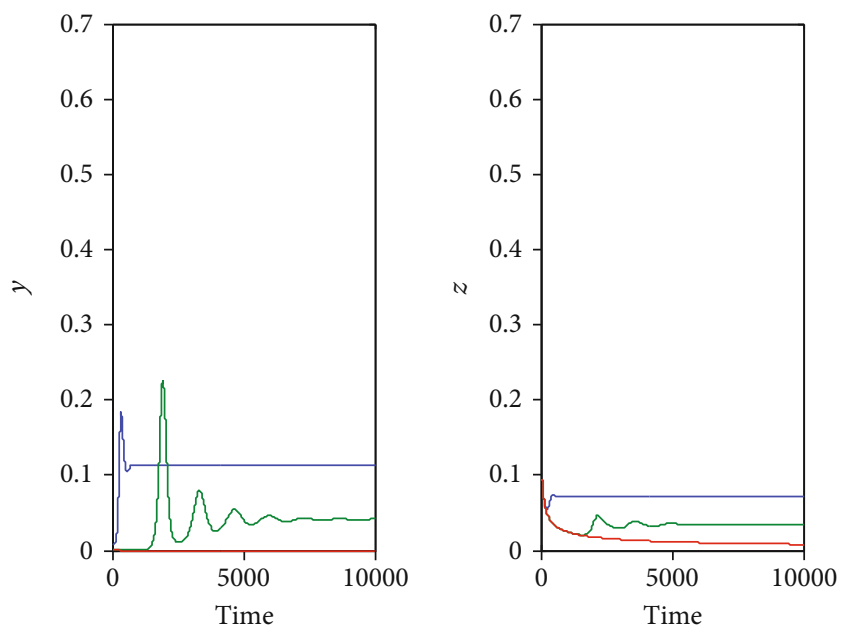

(c)

(d)

FIGURE 3: (a) The trajectories of system (4) for the parameters given by (89) with different values of $\alpha_{3}=0.5,1$, and 5 represented by the colors blue, green, and red, respectively, approach asymptotically to CP for the first two values, while the trajectory approaches to SPFP for the third value. (b) The trajectories of $x$ versus $t$. (c) The trajectories of $y$ versus $t$. (d) The trajectories of $z$ versus $t$.

According to Figure 3, increasing the value of $\alpha_{3}$ causes decreasing in the predator's populations and increasing in prey population gradually; then, the solution of system (4) settled at the SPFP first and then at the FAP. However, increasing $\alpha_{5}$ destabilizes system (4) and the solution persists at periodic dynamics. Moreover, it is observed that the effect of the parameter $\alpha_{4}$ is similar to that of $\alpha_{3}$.
Now, the effects of varying the parameters $\alpha_{6}$ and $\alpha_{9}$ on system (4) dynamical behavior are shown in Figures 5 and 6, respectively.

Obviously, Figures 5 and 6 show that the parameters $\alpha_{6}$ and $\alpha_{9}$ have opposite effects on the dynamic behavior of system (4) so that as increases $\alpha_{6}$ (decreasing $\alpha_{6}$ ), the population of the prey decreases while the populations of the 


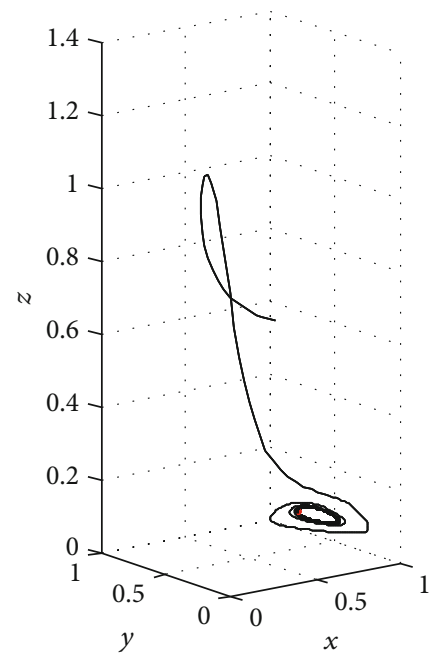

(a)

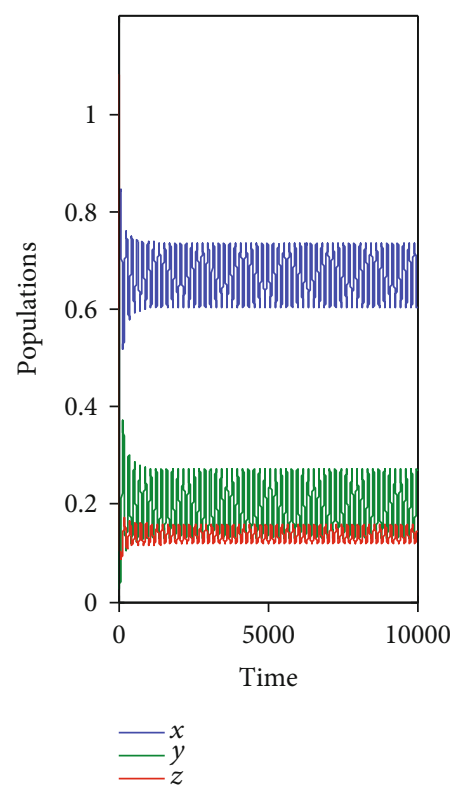

(b)

Figure 4: (a) The trajectory of system (4) approaches asymptotically to periodic dynamics in the positive octant for $\alpha_{5}=20$, with the other parameters as given by (89). (b) Time series for the trajectory given in (a).

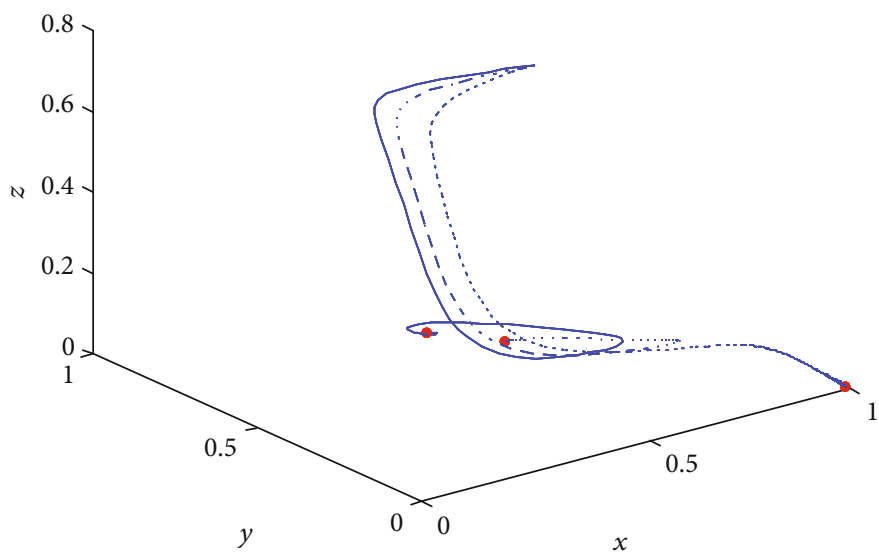

Figure 5: The trajectories of system (4) using the data given by (89) with different values of $\alpha_{6}=0.1,0.5$, and 0.75 , those represented by dotted line, dashed line, and solid line, respectively.

predators increase and hence system (4) loses their persistence and approaches to FAP as decreasing $\alpha_{6}$ (increasing $\left.\alpha_{9}\right)$. Furthermore, it is observed that varying the parameter $\alpha_{10}$ has a quantitative effect on the dynamic behavior of system (4) and system (4) persists at the CP.

The effects of varying the parameters $\alpha_{11}$ and $\alpha_{12}$ on system (4) dynamical behavior are explaining in Figures 7 and 8 , respectively.

According to Figures 7 and 8, it is watched that increasing the parameter $\alpha_{11}$ leads to losing the persistence of the system by approaching the SPFP first and then to SAP. However, decreasing (or increasing) the parameter $\alpha_{12}$ leads to losing the persistence of the system and the solution approaches to GPFP (SPFP). It is observed that the parameter $\alpha_{13}$ has similar effects on the dynamic behavior of system (4) as that for $\alpha_{12}$.

Keeping the above in mind, the second set of parameter values, which leads system (4) to chaotic dynamics, is given in the set of data (90):

$$
\begin{aligned}
& \alpha_{1}=0.8, \alpha_{2}=0.8, \alpha_{3}=4, \alpha_{4}=8, \alpha_{5}=4, \alpha_{6}=1, \alpha_{7}=0.8 \\
& \alpha_{8}=0.25, \alpha_{9}=0.05, \alpha_{10}=0.5, \alpha_{11}=0.1, \alpha_{12}=4, \alpha_{13}=4
\end{aligned}
$$




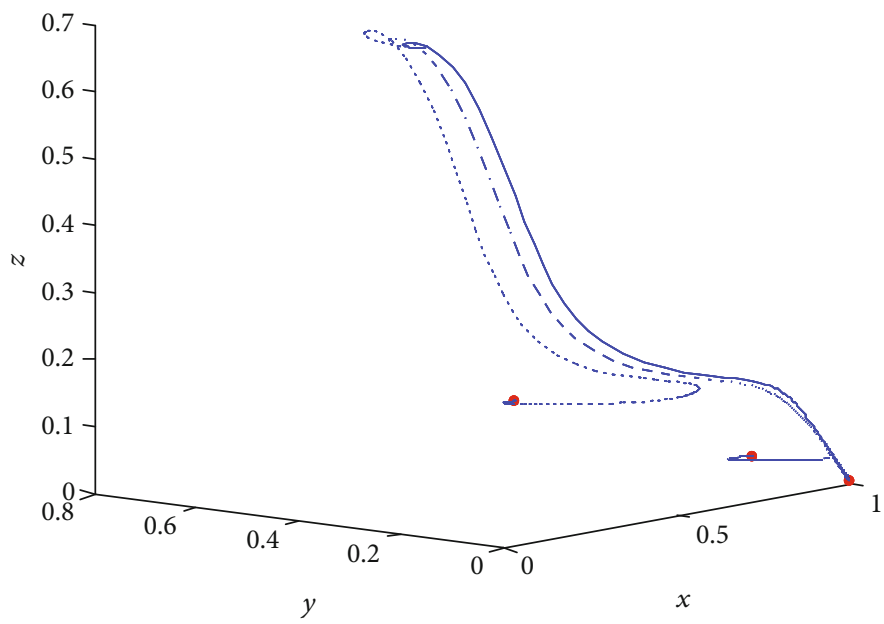

Figure 6: The trajectories of system (4) using the data given by (89) with different values of $\alpha_{9}=0.01,0.15$, and 0.25 , those represented by dotted line, dashed line, and solid line, respectively.

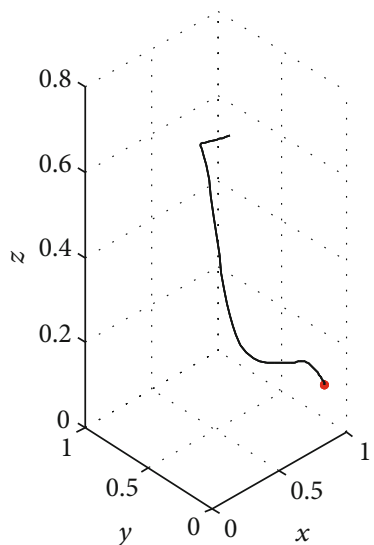

(a)

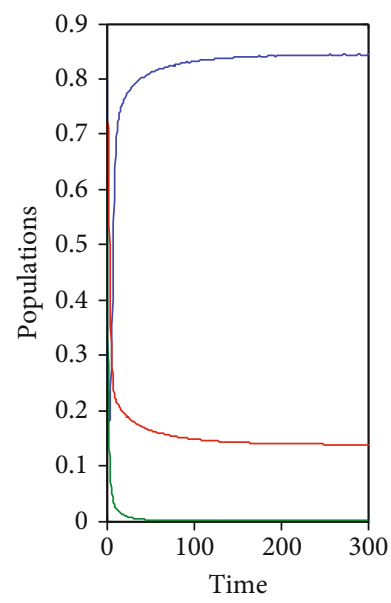

(b)

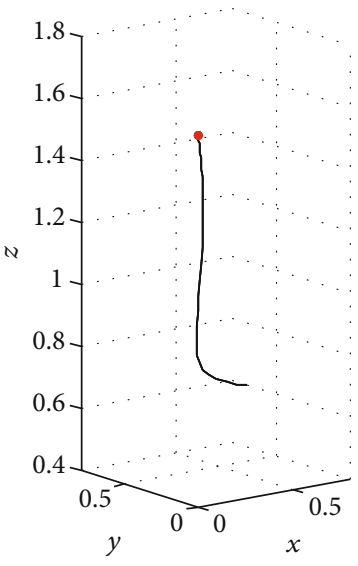

(c)

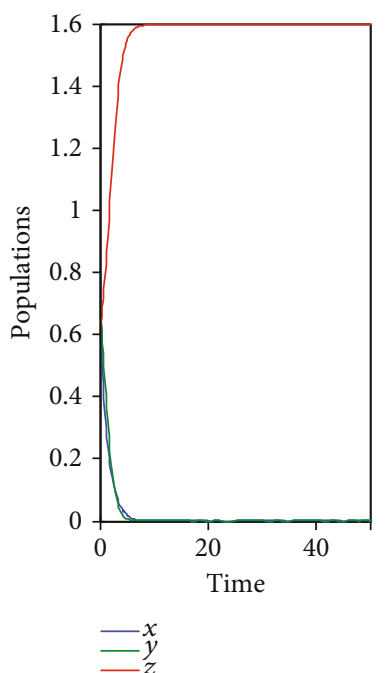

(d)

Figure 7: (a) The trajectories of system (4) using the data given by (89) with $\alpha_{11}=0.24$ approaches asymptotically to SPFP. (b) Time series for the trajectory given in (a). (c) The trajectories of system (4) using the data given by (89) with $\alpha_{11}=1$ approaches asymptotically to SAP. (d) Time series for the trajectory given in (c).

The dynamic behavior of system (4) is shown in Figures 9 and 10.

According to Figures 9 and 10, system (4) is rich in its dynamics including point attractors, periodic attractors, and chaos. It has many bifurcation points when varying its parameter values. It is well known that the most property that characterizes the chaotic attractor is the sensitivity to the varying initial points. So, Figure 11 shows clearly the sensitivity of the strange attractor given in Figure 9 to a small change in their initial point.

Now, the impacts of varying some parameter values on the chaotic regions and the dynamic behavior of system (4) are studied using the bifurcation diagrams. It is known that the bifurcation diagram shows the birth and death of the attractors as a function of varying the parameter values. The bifurcation diagrams (BD) as a function of the parameters $\alpha_{1}, \alpha_{7}, \alpha_{9}, \alpha_{12}$, and $\alpha_{13}$ are shown in Figures 12-16.

According to Figures 12 and 13, there are ranges around $\alpha_{1}=0.8$ and $\alpha_{7}=0.8$ in which the system is chaotic; however, it faces extinction in the specialist predator as these parameters increase and the trajectories approach periodic dynamics in $x z$-plane. Similar effects on the dynamics behavior of system (4) are observed for the parameters $\alpha_{2}$ as those of $\alpha_{1}$ and $\alpha_{7}$. Finally, the other BD are clearly shown the existence of chaotic regions; however, it is watched that 


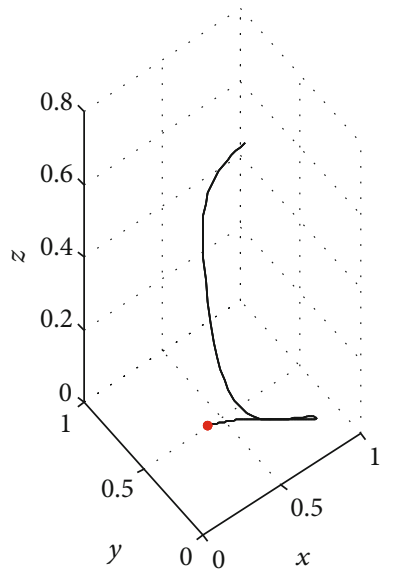

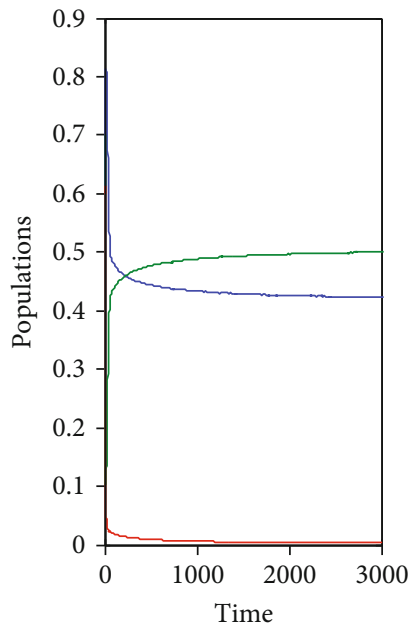

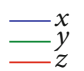

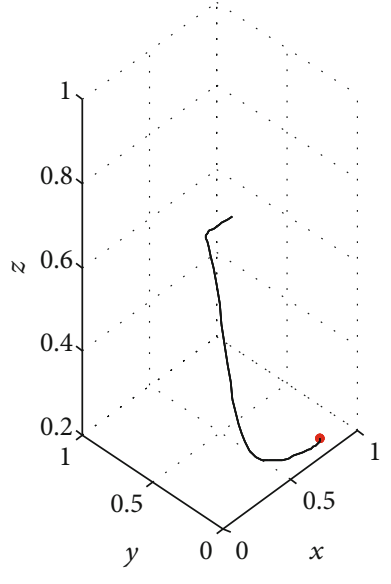

(c)

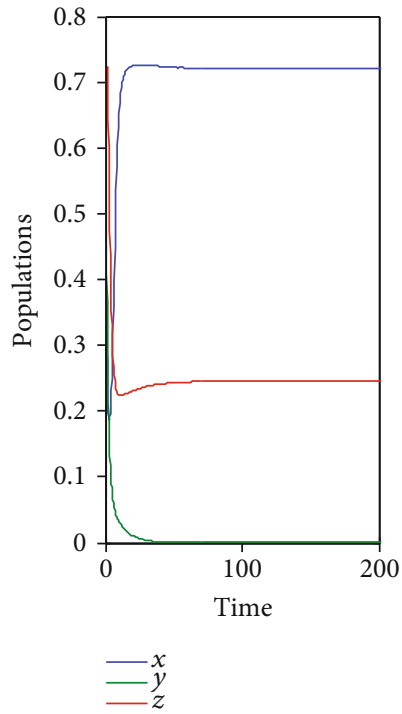

(d)

FIGURE 8: (a) The trajectories of system (4) using the data given by (89) with $\alpha_{12}=0.1$ approaches asymptotically to GPFP. (b) Time series for the trajectory given in (a). (c) The trajectories of system (4) using the data given by (89) with $\alpha_{12}=1.25$ approaches asymptotically to SPFP. (d) Time series for the trajectory given in (c).

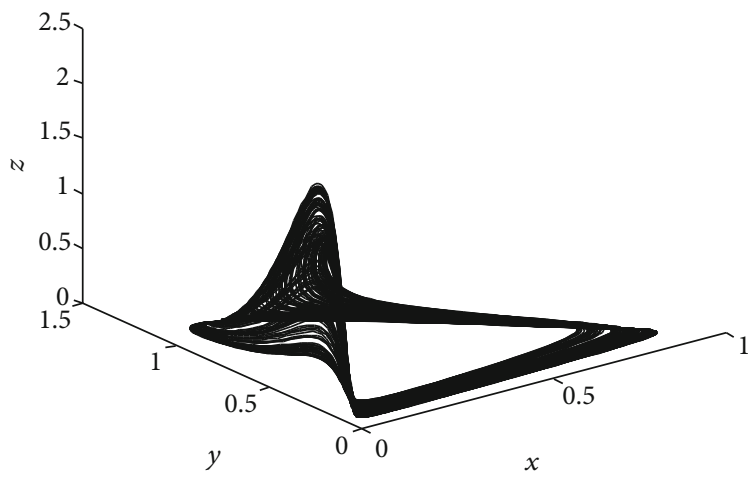

(a)

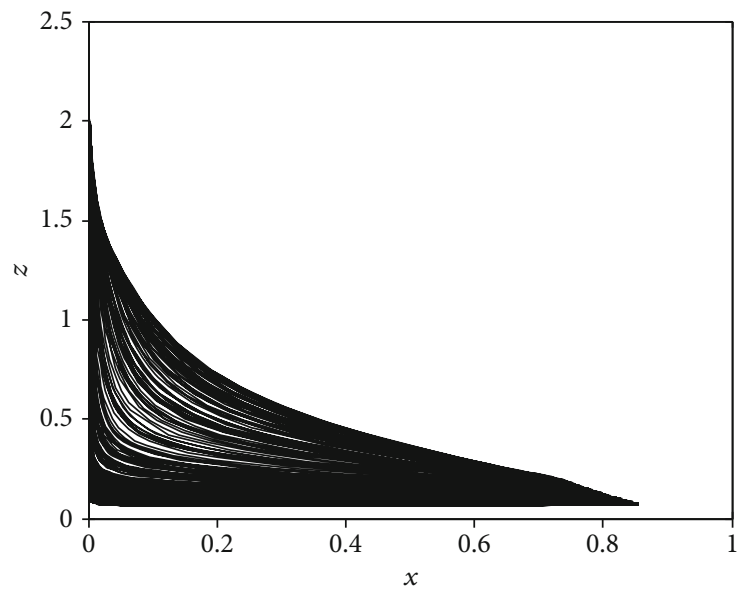

(c)

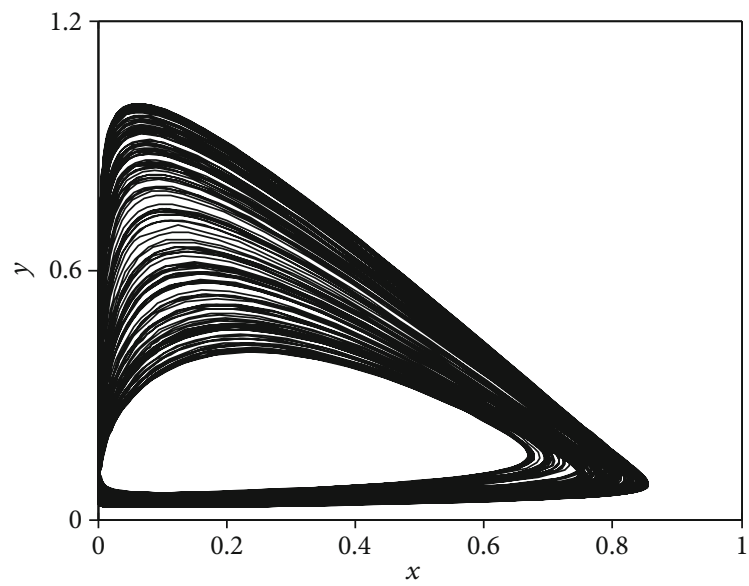

(b)

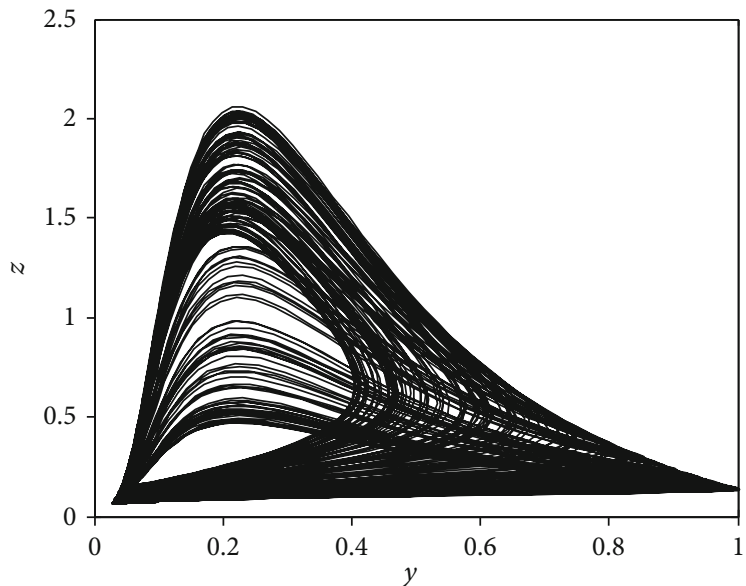

(d)

FIgURE 9: (a) The trajectory of system (4) approaches a chaotic attractor using the dataset given by (90), while their projection on $x y, x z$, and $y z$ planes are shown in (b), (c), and (d), respectively. 

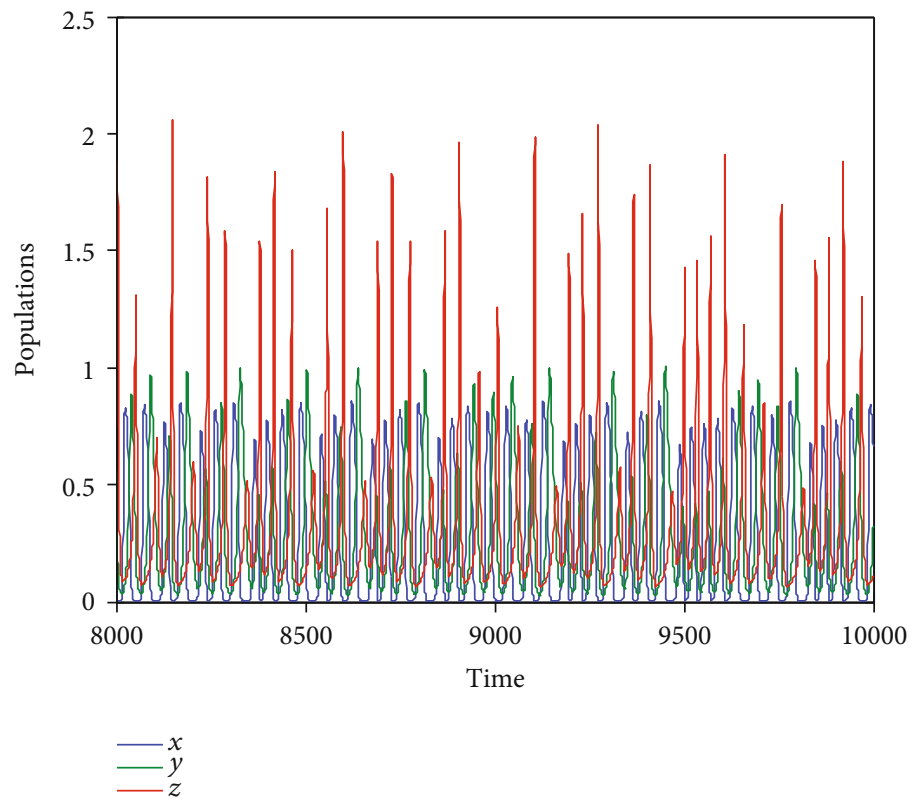

Figure 10: Time series of the chaotic attractors given in Figure 9.

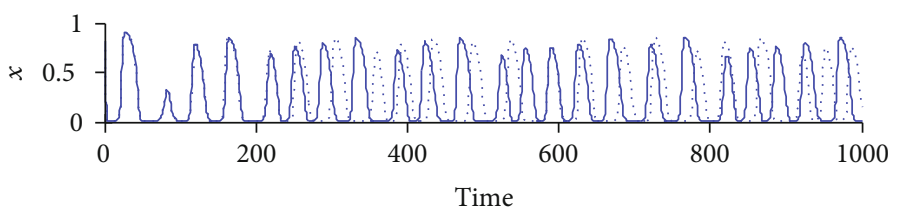

(a)

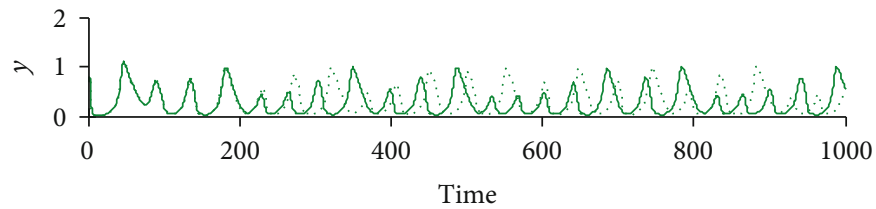

(b)

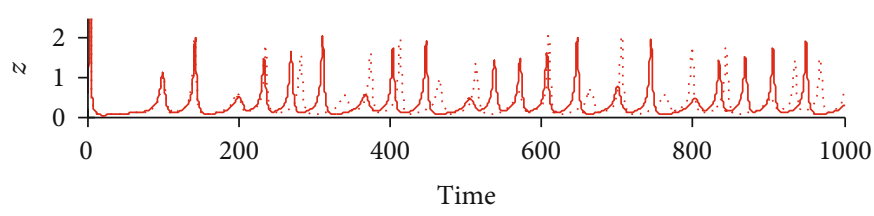

(c)

FIGURE 11: Sensitivity of the trajectory of the chaotic attractor that is given in Figure 9(a) to the initial values. Solid line starts at $(0.8,0.7,0.6)$ while the dotted line starts at $(0.81,0.71,0.61)$. (a) Trajectories of $x$ versus $t$. (b) Trajectories of $y$ versus $t$. (c) Trajectories of $z$ versus $t$.

increasing these parameters leads to extinction in one predator species and the solution approaches to periodic dynamics in the plane.

\section{Discussion and Conclusion}

In this paper, a food-web model having specialist and generalist predators is proposed and studied. It is assumed that the prey has antipredator properties against predation and hence Sokol-Howell functional responses are used to satisfy this assumption. The generalist predator has an alternative food source, and hence, the Leslie-Gower type generalist predator is also included in the system. The impact of fear on the growth rate of the prey due to their fear of predation is also considered. All the properties of the solution of the model are discussed. The equilibria and their stability analysis are studied. The persistence conditions are established. The regions of asymptotic stability of these equilibria are 


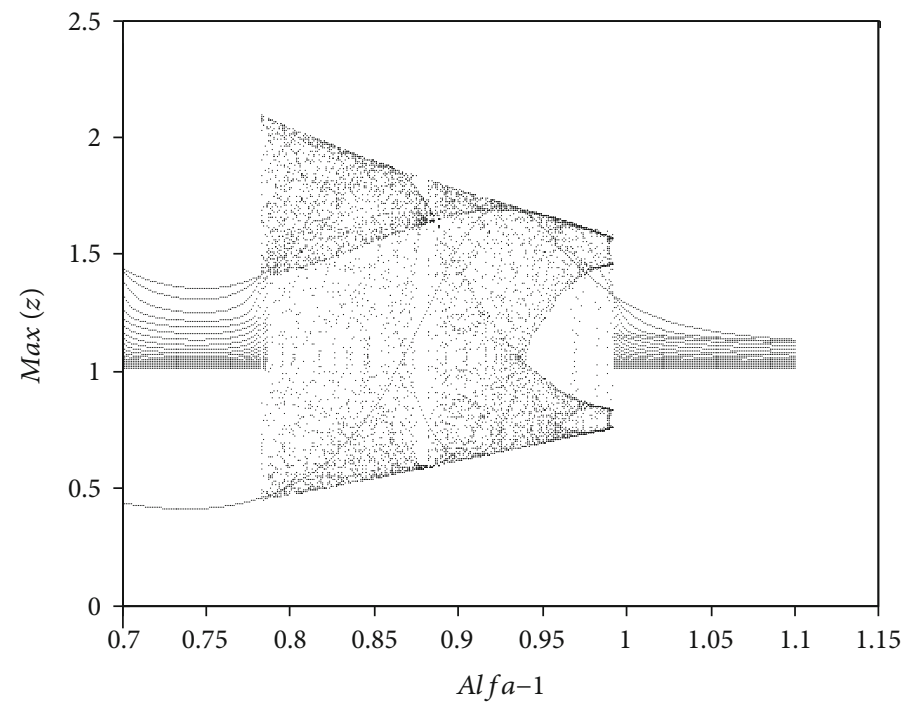

FIgURE 12: The BD of system (4) as a function of $\alpha_{1}$ in the range $0.7<\alpha_{1}<1.1$ using the parameter values in (90).

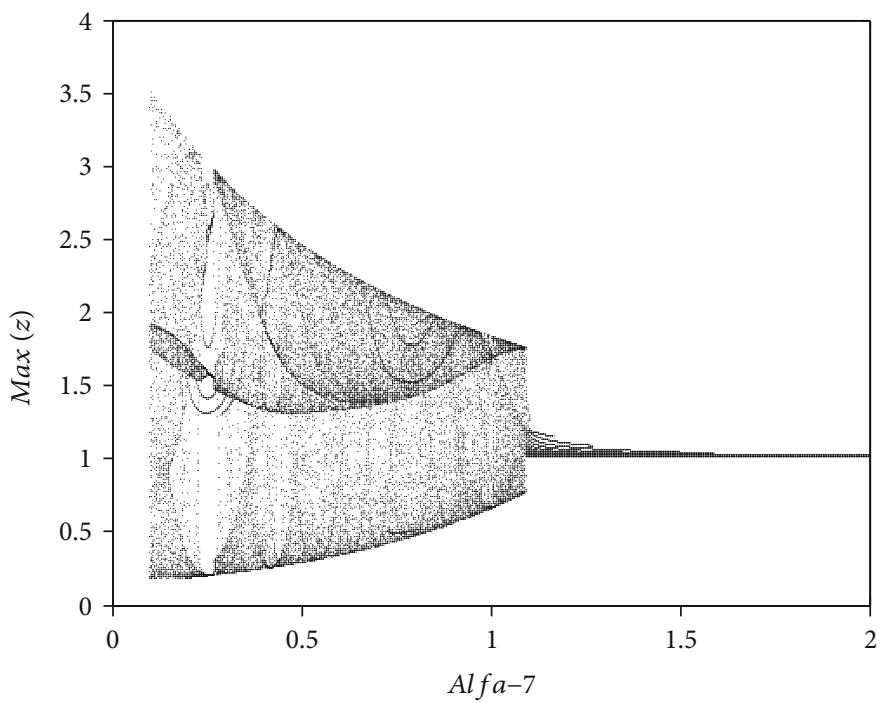

FIGURE 13: The BD of system (4) as a function of $\alpha_{7}$ in the range $0.1<\alpha_{7}<2$ using the parameter values in (90).

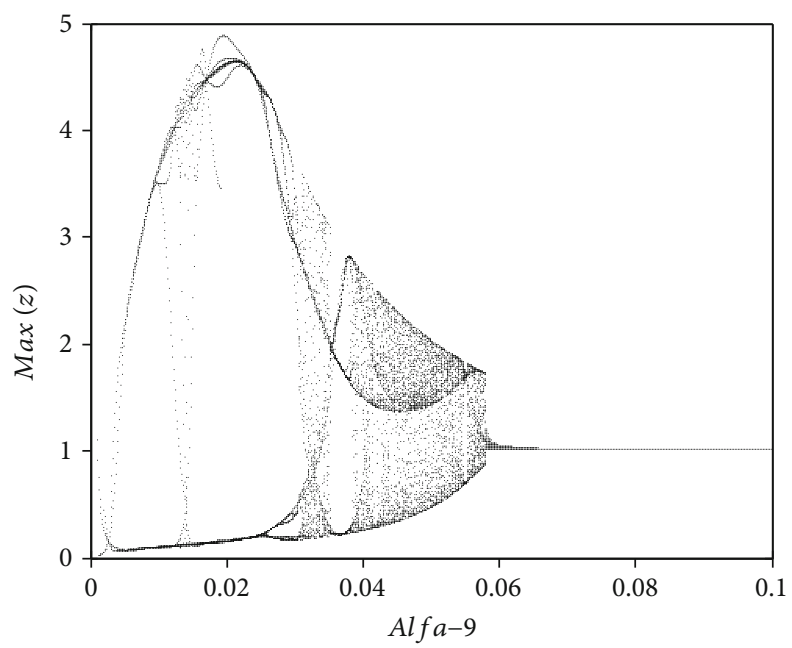

FIgURE 14: The BD of system (4) as a function of $\alpha_{9}$ in the range $0.01<\alpha_{9}<0.1$ using the parameter values in (90). 


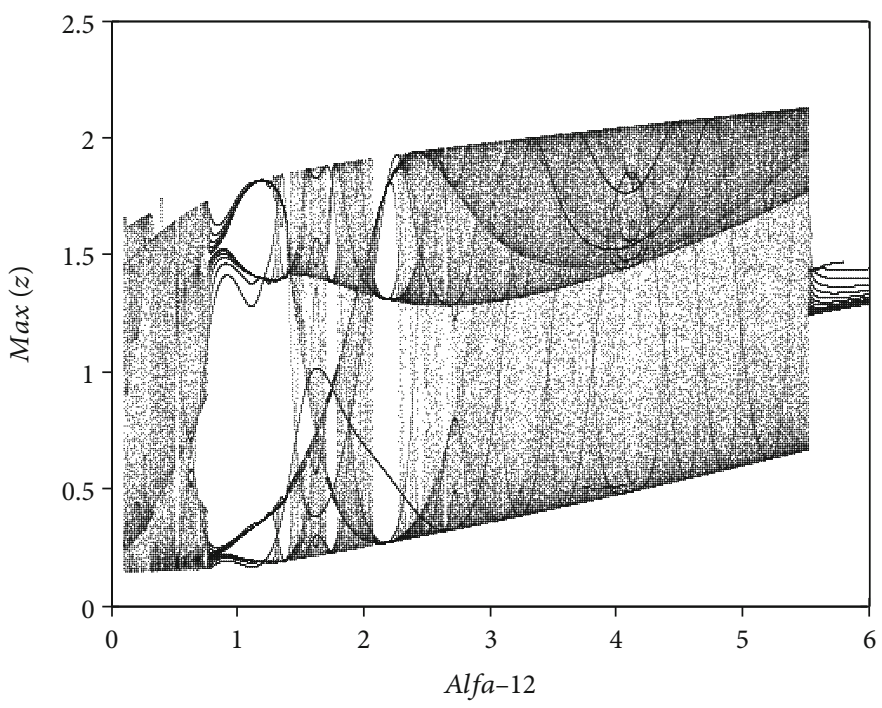

FIGURE 15: The BD of system (4) as a function of $\alpha_{12}$ in the range $0.01<\alpha_{12}<6$ using the parameter values in (90).

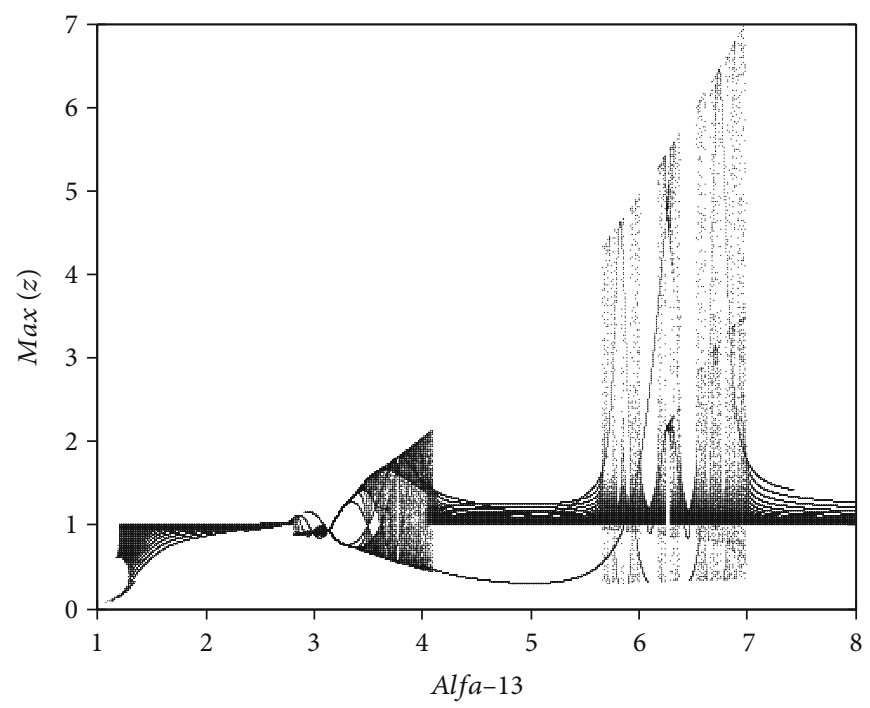

FIgURE 16: The $\mathrm{BD}$ of system (4) as a function of $\alpha_{13}$ in the range $1<\alpha_{13}<8$ using the parameter values in (90).

determined with the help of the Lyapunov method. Local bifurcation is investigated whenever it exists. Finally, extensive numerical simulations are performed to understand the effects of varying the parameter values on the dynamics of the system using two sets of parameters. It is watched that system (4) is wealthy in its dynamical behavior including all possible attractors' types such as point attractor, periodic attractor, and chaos. The fear coefficients have a clear control on the dynamics of the system so that as the fear increases, the chaotic regions decrease and the solution settled to $\mathrm{CP}$ or periodic dynamics in the boundary planes. Increasing the preference rates, which are equivalent to increasing the prey's group defense, leads to loss of the system persistence and the solution approaches asymptotically to FAP. Increasing the death rate of the specialist predator causes extinction in both the predators and the system approaches to FAP. Finally, system (4) is very sensitive to changes in the abundance of alternative food sources.

\section{Data Availability}

All supportable data are given in the main text.

\section{Conflicts of Interest}

The authors declare that there are no conflicts of interest. 


\section{Acknowledgments}

This article is funded by the authors themself only.

\section{References}

[1] C. S. Holling, "The components of predation as revealed by a study of small-mammal predation of the European pine sawfly," Canadian Entomologist, vol. 91, no. 5, pp. 293-320, 1959.

[2] F. Hua, K. E. Sieving, R. J. Fletcher, and C. A. Wright, "Increased perception of predation risk to adults and offspring alters avian reproductive strategy and performance," Behavioral Ecology, vol. 25, no. 3, pp. 509-519, 2014.

[3] E. L. Preisser and D. I. Bolnick, "The many faces of fear: comparing the pathways and impacts of non-consumptive predator effects on prey populations," PLoS One, vol. 3, no. 6, article e2465, 2008.

[4] J. P. Suraci, M. Clinchy, L. M. Dill, D. Roberts, and L. Y. Zanette, "Fear of large carnivores causes a trophic cascade," Nature Communications, vol. 7, no. 1, article 10698, 2016.

[5] X. Wang and X. Zou, "Modeling the fear effect in predatorprey interactions with adaptive avoidance of predators," Bulletin of Mathematical Biology, vol. 79, no. 6, pp. 1325-1359, 2017.

[6] R. Upadhyay and S. Mishra, "Population dynamic consequences of fearful prey in a spatiotemporal predator-prey system," Mathematical Biosciences and Engineering, vol. 16, no. 1, pp. 338-372, 2018.

[7] P. Panday, N. Pal, S. Samanta, and J. Chattopadhyay, "Stability and bifurcation analysis of a three-species food chain model with fear," International Journal of Bifurcation and Chaos, vol. 28, no. 1, article 850009, 2018.

[8] A. Das and G. Samanta, "Modeling the fear effect on a stochastic prey-predator system with additional food for the predator," Journal of Physics A: Mathematical and Theoretical, vol. 51, no. 46, article 465601, 2018.

[9] V. Kumar, N. Kumari, and School of Basic Sciences, Indian Institute of Technology Mandi, Mandi, Himachal Pradesh, 175005, India, "Controlling chaos in three species food chain model with fear effect," AIMS Mathematics, vol. 5, no. 2, pp. 828-842, 2020.

[10] M. A. Aziz-Alaoui, "Study of a Leslie-Gower-type tritrophic population model," Chaos, Solitons and Fractals, vol. 14, no. 8, pp. 1275-1293, 2002.

[11] N. Zhang, C. Fengde, S. Qianqian, and W. Ting, "Dynamic behaviors of a harvesting Leslie-Gower predator-prey model," Discrete Dynamics in Nature and Society, vol. 2011, Article ID 473949, 14 pages, 2011.

[12] C. Yongli, Z. Caidi, and W. Weiming, "Spatiotemporal complexity of a Leslie-Gower prey-predator model with the weak Allee effect," Journal of Applied Mathematics, vol. 2013, Article ID 535746, 16 pages, 2013.

[13] W. Abid, R. Yafia, M. A. Aziz-Alaoui, and A. Aghriche, "Dynamics analysis and optimality in selective harvesting predator-prey model with modified Leslie-Gower and Holling-type II," Nonautonomous Dynamical Systems, vol. 6, no. 1, pp. 1-17, 2019.

[14] K. M. Singh and B. S. Bhadauria, "Qualitative analysis of a modified Leslie-Gower prey-predator model with weak Allee effect," Applications and Applied Mathematics: An International Journal, vol. 16, no. 1, pp. 163-190, 2021.
[15] W. Sokol and J. A. Howell, "Oxidation of an inhibitory substrate by washed cells (oxidation of phenol by Pseudomonas putida)," Biotechnology and Bioengineering, vol. 30, no. 8, pp. 921-927, 1987.

[16] R. K. Upadhyay, S. R. K. Iyengar, and V. Rai, "Chaos: an ecological reality?," International Journal of Bifurcation and Chaos, vol. 8, no. 6, pp. 1325-1333, 1998.

[17] R. K. Upadhyay, V. Rai, and S. R. K. Iyengar, "Species extinction problem: genetic vs ecological factors," Applied Mathematical Modelling, vol. 25, no. 11, pp. 937-951, 2001. 